\title{
Boundary-layer approaches to dendritic growth
}

\author{
John D. Weeks and Wim van Saarloos \\ AT\&T Bell Laboratories, Murray Hill, New Jersey 07974-2070
}

(Received 24 September 1986)

\begin{abstract}
We analyze the derivation of boundary-layer models for dendritic growth and investigate the extent to which they yield information about the existence of a continuous family of steady-state needle crystal solutions. Although recent work has established that there exists only a discrete set of solutions for viscous fingering in a Hele-Shaw cell, side walls play an important role in this system, and we argue on physical grounds that the same mechanism may not apply to free dendrites. After a discussion highlighting the physical differences in these two systems, we analyze the model equations for dendritic growth, which first suggested the breakup of the family. We develop a systematic, and in principle exact, boundary-layer formalism for diffusion-controlled dendritic growth starting from the full heat-conduction equation. A consistent application of the formalism generates an expansion of the smooth steady-state solutions in powers of $(1-\Delta)$, where $\Delta$ is the dimensionless undercooling, but gives no indication as to whether or not a family of such solutions exist. Different physically motivated approximations yield different model equations, including the boundary-layer model of Ben-Jacob and co-workers, with very different properties. Steady-state predictions of all such models are arbitrary. We show that a proper phase-space description requires an infinitedimensional phase space, in which there are stable directions not found in the boundary-layer model.
\end{abstract}

\section{INTRODUCTION}

\section{A. Heat-conduction equation}

Recently there has been much interest in dendritic growth, both as a challenging problem in material science and crystal growth, and more generally as an important example of pattern selection in nature. ${ }^{1}$ Experiments have shown that the tip of a dendrite, freely growing into an undercooled melt, advances uniformly with a fixed velocity $V_{t}$. The tip curvature $\kappa_{t}$ and side-branch spacings seem to be independent of initial transients in the growth process, and depend only on the undercooling., ${ }^{2,3}$ Yet theoretical understanding of even this basic fact is far from complete.

The physics of dendritic growth is controlled by the diffusion of the latent heat of crystallization away from the interface. Let us consider the simplest case of a twodimensional one-component system in the "one-sided" limit where we neglect heat diffusion in the solid. ${ }^{1,4}$ The growth rate is determined by the dimensionless undercooling $\Delta$, defined to be the difference between the bulk melting temperature and the temperature of the melt far in front of the tip, measured in units of $L / c$, the ratio of the latent heat to the specific heat. In most experiments, ${ }^{3}$ we have $\Delta \ll 1$. If we consider dimensionless temperatures measured relative to that of the melt (so that the bulk melting temperature is $\Delta$ ), the dimensionless temperature field $T(x, z, t)$ satisfies the heat-flow equation

$$
\frac{\partial T}{\partial t}=D \nabla^{2} T
$$

together with appropriate boundary conditions. Here $(x, z)$ are Cartesian coordinates in the lab frame, $t$ is the time, and $D$ is the thermal diffusivity. Far from the in- terface we have $T \rightarrow 0$ and at the interface, assuming only small departures from local equilibrium, we required that the interface temperature $T_{i}$ satisfy

$$
T_{i}=\Delta-d_{0} \kappa_{i}-\mu V_{n} .
$$

The first two terms on the right give the equilibrium melting temperature $T_{i}^{\text {eq }}$ of an interface with curvature $\kappa_{i}$, given by the Gibbs-Thomson relation ${ }^{1}$

$$
T_{i}^{\mathrm{eq}}=\Delta-d_{0} \kappa_{i} .
$$

Here $d_{0}$ is the capillary length, ordinarily of order angstroms, which is proportional to the surface tension. The last term on the right in (1.2) takes account of interface kinetics and assumes a linear relationship between the normal interface velocity $V_{n}$ and the effective interface undercooling $T_{i}^{\mathrm{eq}}-T_{i}$. Both $\mu$ and $d_{0}$ can depend on crystalline orientation; frequently $\mu$ is set equal to zero in theoretical studies, and we will do so here unless otherwise indicated. ${ }^{5}$ Finally, the release of latent heat at the interface as the crystal grows is taken into account by the heat-conservation relation

$$
V_{n}=-D(\widehat{\mathbf{n}} \cdot \nabla T)_{i},
$$

where $\hat{\mathbf{n}}$ is the unit normal pointing into the melt. Equation (1.4) equates the rate of heat production at the interface to heat flow into the melt.

Before considering more complicated time-dependent problems, it is natural to seek steady-state solutions of (1.1)-(1.4). A growing dendrite, constantly emitting side branches, clearly is not in a steady state, but such solutions could have relevance for the motion of dendrite tips, particularly if one assumes that only some time-averaged shape is important in determining the temperature field near the tip. See Fig. 1(a). However, it is not obvious that 


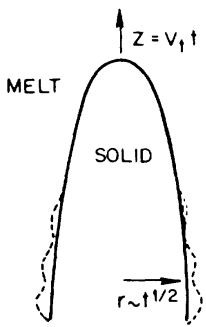

(a)

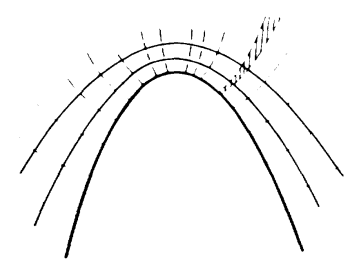

(b)
FIG. 1. Dendrites and needle crystals. (a) The dendrite tip viewed as a superposition of a steady-state needle solution (solid line) and the side-branching instability (dashed lines). (b) A needle crystal (heavy line) and associated isotherms (light lines). A tube normal to the interface as pictured in the BLM is indicated by the dashed region.

this is the case, and we study the steady-state problem only as the first step leading towards a full dynamical treatment.

Unfortunately, such is the complexity of the heat-flow equations (1.1)-(1.4) that even in the steady state an exact solution has been found only in the artificial "Ivantsov limit," where both $d_{0}$ and $\mu$ are set equal to zero in (1.2). In this approximation the growing interface always maintains the bulk melting temperature. The Ivantsov solutions $^{6,7}$ are parabolas moving at a constant velocity $V$ in the $z$ direction,

$$
(z-V t)+\frac{\kappa_{t}}{2} x^{2}=0,
$$

where the growth velocity $V$ and tip curvature $\kappa_{t}$ are related to the undercooling by only a single equation of the form

$$
p^{-1} \equiv \frac{2 D \kappa_{t}}{V}=f(\Delta)
$$

and $f(\Delta)$ is a known function of the undercooling $\Delta$. Here $p$, the Péclet number, gives the radius of curvature of the tip in units of the diffusion length $2 D / V$. Though dendritic tips indeed appear parabolic and move at a constant velocity, the Ivantsov result (1.6) predicts the existence of a continuous family of solutions, ranging from fat slow-growing shapes to sharp rapidly growing ones, at the same undercooling $\Delta$. This disagrees with the experimental result ${ }^{2,3}$ that tips with a single $\kappa_{t}$ and $V_{t}$ are found for a given $\Delta$.

It was recognized long ago that this discrepancy could arise from the Ivantsov boundary condition $T_{i}=\Delta$. Indeed with $d_{0}$ and $\mu$ set equal to zero, there is only one length $D / V$ in the problem and a scaling like (1.6) must be found. ${ }^{1,7}$ However, since the corrections to $T_{i}$ from $d_{0}$ and $\mu$ in Eq. (1.2) are usually very small, it was generally assumed that a family of steady-state "needle crystal" solutions slightly perturbed from the Ivantsov family continued to exist, and that some kind of additional (dynamical) selection mechanism was needed to pick out the solution actually observed in experiment. The most successful approach based on this idea was the marginal-stability hypothesis of Langer and Müller-Krumbhaar, ${ }^{8,1}$ which accurately represented the experimental data, though fundamental justification was lacking.

Recently two groups ${ }^{9,10}$ proposed simple model equations for the motion of the interface that were designed to mimic several features of the full problem (1.1)-(1.4), while remaining simple enough to permit exact analysis and numerical simulation. These models express the velocity at any point of the interface in terms of locally defined quantities like the curvature, and we will refer to them as local models. The model equations successfully reproduced the existence of a family of needle crystal solutions in the Ivantsov limit. Analysis showed that in both the geometric model (GM) of Brower et al. ${ }^{9}$ and in the boundary-layer model (BLM) of Ben-Jacob et al., ${ }^{10}$ the $d_{0}$ term in (1.2) and (1.3) is a singular perturbation, in whose presence at most a discrete set of steady-state solutions can survive. Indeed in the absence of crystalline anisotropy, no steady-state solutions at all exist with $d_{0}$ nonzero for these models. Of course, as all these workers realized, the models' results only indicated the possibility that such behavior can occur in the full nonlocal problem. Nevertheless, very recent analysis of the full problem (see below) seems to be in agreement with these ideas.

As will be discussed in more detail below, the model equations suggest that there is in general a problem in finding solutions with smooth behavior at the tip of the needle that also join onto the Ivantsov-like needle solutions far down in the tails of the needle. Crystalline anisotropy ${ }^{11}$ is needed to provide a proper matching of physically acceptable tip and tail behavior; even then the resulting smooth needlelike solutions exist only at a discrete set of velocities. In effect the steady-state problem itself with the proper boundary condition (1.3) provides most of the "selection," with the discrete solution of maximum velocity actually being observed in numerical simulations., 9 Since it is the capillary length that singles out particular discrete solutions from a seemingly continuous family as it shows up in a naive perturbation theory, this mechanism is often termed "microscopic solvability."

This scenario has recently been shown to apply to the problem of viscous fingering in a Hele-Shaw cell, ${ }^{12-14}$ whose mathematical description has several features in common with dendritic growth, and methods similar to those used to solve the viscous fingering problem seem to confirm that microscopic solvability also applies to dendritic growth. ${ }^{15}$ Finally, there exist numerical simulations of the full steady-state needle crystal problem that again seem consistent with this scenario. ${ }^{16,17}$

Despite this, we believe that the steady-state equations (1.1)-(1.4) for dendritic growth have a number of physical and mathematical properties that one could intuitively associate with the general existence of a continuous family of steady-state solutions. ${ }^{18}$ Although we can provide no rigorous analysis of this delicate mathematical question, these ideas are worth exploring to help determine what physical properties decide between one scenario or the other and to gain a physical understanding of the solvability condition if it applies to dendrites.

In this paper we will confine ourselves to a critical as- 
sessment of the model equations, which first suggested the possibility of the microscopic-solvability mechanism. A full discussion of the more recent numerical and analytical work regarding this problem will be given in another paper where we present other results of our own, ${ }^{19}$ but we briefly summarize our views on some of these questions in Sec. III. The main question we address in this paper is the degree to which the model equations can be systematically derived from the full heat-flow equations (1.1)-(1.4). In particular, how reliable are the predictions of a given model equation concerning the existence of steady-state solutions? The formalism we develop for treating the moving interface and the associated temperature (and other) fields, while particularly incisive in assessing the model equations, may also prove useful in other more general applications to moving-boundary problems and in determining the dynamic relevance of steady-state considerations in general.

Although many of the issues raised in our study of the model equations are of general interest and have broad implications, some of the mathematics is rather technical. For this reason we conclude this section with a physical discussion of our viewpoint on dendritic growth, and in Sec. II provide a general introduction to the main points we will raise in the rest of the paper concerning the model equations. Section III briefly discusses other numerical and analytical work that appears to confirm the microscopic-solvability scenario for dendritic growth and our belief that some important questions may remain unsettled. Sections IV-IX provide the details of our work with most mathematical points contained in several appendixes.

\section{B. Physical picture}

Before beginning a detailed discussion of the models, we first sketch our physical picture of diffusion controlled growth. This picture, based in part on ideas introduced by Ben-Jacob et al., ${ }^{10}$ underlies our reservations about the microscopic-solvability scenario as applied to dendritic growth. Growth of crystals at small undercoolings is dominated by the necessity to transport the latent heat produced at the interface into the cooler liquid by a slow diffusion process. Since there are no sources of heat loss (heat sinks) in Eqs. (1.1)-(1.4), the heat produced at the moving interface is conserved and can be directly calculated from the temperature field. The heat content of a unit volume of liquid relative to that of the unheated melt in units of $L$ is directly given by (the dimensionless quantity) $T$.

A rough measure of the amount of heat in front of the interface can be obtained from the boundary-layer thickness $l$, defined with the help of Eq. (1.4) as

$$
l \equiv \frac{D T_{i}}{V_{n}} .
$$

Here $l$ is the distance over which the temperature (heat content) in front of the interface falls to its asymptotic value as determined by a linear extrapolation of the normal temperature gradient at the interface. ${ }^{10,20}$ Then $H$, the total heat content per unit length of interface con- tained in a "tube" normal to the interface, is approximately given by $H \approx T_{i} l . \quad H$ is a fundamental quantity in the BLM of Ben-Jacob et al. ${ }^{10}$ and we will later give a precise definition. See Fig. 1(b).

Consider first the growth of a planar interface. Only the small fraction $T_{i}$ of the latent heat released when a unit volume crystallizes could be accommodated by heating material ahead of the interface if the interface were to advance with an undistorted temperature profile. Therefore the remaining latent heat fraction $\left(1-T_{i}\right)$, the heat surplus, must increase $l$ and the heat content in the boundary layer. From (1.7) this reduces the growth velocity $V_{n}$. The growth rate is limited by the rate at which the heat surplus can diffuse away. The process is clearly nonlocal in time; heat released at earlier times still affects the present growth rate. Not surprisingly, there is a typical diffusion relation between the growth velocity $V_{n}$ and the time $t: V_{n} \sim t^{-1 / 2}$. At long times the growth rate of a planar interface tends to zero as heat continually piles up in front in the boundary layer. ${ }^{1,10}$

The physics is very different at undercooling $\Delta$ so large that $\Delta \geq 1$. Then the heat surplus vanishes and steadystate growth of a plane is possible when interface kinetics is taken into account., ${ }^{1,21}$ For mathematical convenience we will later examine large undercoolings $\Delta \rightarrow 1^{-}$for which the heat surplus is nonzero but very small. This limit preserves many features of the more physically relevant small undercooling case (in particular, the $t^{-1 / 2}$ behavior is still found), but it also permits expansions in powers of $(1-\Delta)$ and is the most favorable limit for examining the use of local-model equations. However, we should not forget the possibility that the physical picture-and selection mechanism-appropriate for large undercoolings could differ from that found at small undercoolings.

When there is a nonzero heat surplus (i.e., $\Delta<1$ ), more rapid growth can occur only if the constraint of planarity is relaxed. Indeed the planar interface is unstable. Part of the heat surplus that is generated by an outward bulge of the solidification front into the liquid can be diverted to the sides. This permits a larger temperature gradient in the forward direction and hence from (1.4) leads to more rapid growth. This is the physical origin of the MullinsSekerka instability, ${ }^{22}$ which underlies the complicated solidification patterns formed by dendrites. ${ }^{23}$

Just as is the case for the initial response leading to the Mullins-Sekerka instability, a needlelike shape permits more rapid long-time growth in the forward direction by directing the heat surplus to the sides. The sharper the curvature of the needle, the more effective is this diversion, and the more rapidly can the needle grow. ${ }^{24}$ Of course, growth along the sides of the needle must then slow down. In this way we can understood the Ivantsov result (1.5) for a steady-state family of moving parabolas. At fixed $z$, the growth rate in the $x$ direction (essentially normal to the interface in the tails of the needle) slows down as $t^{-1 / 2}$, just as for the growing plane, as the heat surplus piles up. The needle achieves a constant growth rate in the $z$ direction at the cost of slow growth in the $x$ direction. See Fig. 1(a). The redirected heat surplus in the boundary layer causes a natural joining of the 
behavior near the tip to that in the tails.

More generally, we expect the tails to lock into the asymptotic Ivantsov-like $V_{n} \sim t^{-1 / 2}$ behavior, even in the presence of (small) temperature perturbations near the tip; the buildup of heat in the boundary layer makes it impossible for the asymptotic behavior to be either faster or slower, and serves to suppress any divergent response. Thus crystalline anisotropy, kinetic undercooling, or capillary corrections should not affect stability deep in the tails. In effect, the Ivantsov-like behavior in the tails resembles an attractive fixed point, towards which several trajectories (generated by different perturbations at the tip) flow. We have already given a local-model equation that exhibits this behavior, ${ }^{18}$ and will discuss it in detail later in Sec. VII. Evidence for this behavior in the full heat-flow equations at distances larger than the diffusion length $2 D / V$ from the tip will be presented elsewhere. ${ }^{19}$ This picture differs greatly from that predicted by the BLM and GM equations. There the structure in the tails is very "fragile," and only a single trajectory flows into the Ivantsov-like fixed point. This causes nontrivial matching conditions between smooth behavior in the tip and in the tails, and in general no steady-state solutions exist. 9,10

Of course, one should not forget that the physical problem concerns the motion of dendrite tips, and any such matching should really be between the tip and some intermediate region before which side branching becomes significant. Experimentally, at small undercoolings, this occurs in a region of order the radius of curvature of the selected tip. ${ }^{1}$ It is here that the physical processes leading to selection must occur. Although the model equations suggest that a study of stability deep in the tails gives information relevant to the selection process, this may not be the case for the full problem. A nonlocal analysis is needed to resolve the remaining questions concerning the validity of the matching picture for the full problem. If it turns out that a family of solutions can be found in a certain parameter range, then an appropriate focus for selection is some dynamic mechanism involving the tip and the intermediate region. Moreover, such an analysis would probably still capture the essential physics in the event that there do exist matching conditions, but which only constrain behavior far down in the (physically irrelevant) tails of the needle.

Our description of the tails relies heavily on the dynamics of heat flow appropriate to a diffusion equation and does not apply to the case of viscous fingering in a HeleShaw cell, where the pressure field satisfies Laplace's equation. $^{25}$ Although the initial Mullins-Sekerka instability is qualitatively the same when the diffusion equation is replaced by Laplace's equation, the long-time (steadystate) behavior is very different. Indeed there are no nonplanar steady-state solutions at all in the absence of sidewalls in the Hele-Shaw cell. The long-ranged Green's function for the Laplace equation implies a sensitive and instantaneous relationship between the pressure at the interface and at the side walls. Thus it is not too surprising that there could be a singular response to seemingly small interface perturbations in such a system. In contrast, the boundary condition the needle crystal temperature field must satisfy at infinity $\left(T_{\infty} \rightarrow 0\right)$ is that naturally produced by the diffusive dynamics of heat flow, and it imposes no constraint.

These differences arise because the heat-flow equations with the $\partial T / \partial t$ term describe the motion of a compressible "heat fluid" whose density is proportional to the temperature. $^{26}$ Since the fluid is compressible, the heat flux produced at the interface will be absorbed by a change in temperature (heat density) in front of the interface; at distances much larger than the boundary layer thickness $l$, we find the undisturbed melt. On the other hand, the Laplace equation appropriate for the Hele-Shaw cell describes the flow of an incompressible fluid; ${ }^{27}$ fluid flow at the interface implies an instantaneous response and flow at the boundaries. This brings about a rigidity and hence fragility to possible steady-state solutions not present in the compressible and dissipative heat flow found in the dendritic needle crystal problem.

Indeed, the mathematical analogy between viscous fingering and dendrites is even closer if the finite value of the compressibility modulus $k$ of the viscous fluid in the Hele-Shaw cell is taken into account, so that $\partial P / \partial t$ $=-k \nabla \cdot V$ with $P$ the pressure and $V$ the fluid velocity. Combining this with Darcy's law, ${ }^{25}$ we see that the pressure fluid satisfies a diffusion equation $\partial P / \partial t=D_{\mathrm{HS}} \nabla^{2} P$, where $D_{\mathrm{HS}}=k h^{2} / 12 \mu$ and $h$ the cell height and $\mu$ the viscosity. However, using typical values for viscous fluids used in the cell, we find $D_{\mathrm{HS}} \approx 100 \mathrm{~m}^{2} / \mathrm{sec}$. The diffusion length $2 D_{\mathrm{HS}} / V_{f} \approx 200 \mathrm{~km}$, for a typical finger velocity of $1 \mathrm{~mm} / \mathrm{sec}$, is so large that the cell side walls are always well within the diffusion length, and the use of the Laplace equation is very well justified.

For typical dendrite tips, on the other hand, the diffusion length is of order $0.1 \mathrm{~mm}$, and while this is much larger than the scale of the structure near the tip, it is notably smaller than the cell size or the spacings of primary tips. In a sense, then, viscous fingering is more local than dendrites because of the close presence of the cell side walls well within the diffusion length, and the elimination of all temporal nonlocal effects. ${ }^{27}$ There is a considerable physical difference between the small Péclet numbers appropriate to dendrites and the "zero-Péclet-number limit" that yields the Laplace equation. From this point of view, directional solidification ${ }^{1}$ represents an intermediate case in which the temporal nonlocality implied by the timederivative term in the diffusion equation is not such an essential feature as in the needle crystal problem.

\section{GENERAL REMARKS ON THE MODEL EQUATIONS}

The local models introduced by Ben-Jacob et al. ${ }^{10}$ and Brower et al. ${ }^{9}$ have been very important in suggesting possible mathematical mechanisms that could operate in the full problem, and in serving as a testing ground for new numerical and analytical methods. In addition, the BLM of Ben-Jacob et al. ${ }^{9}$ is very appealing in that it incorporates much of the physics of dendritic growth. These aspects give the BLM a significance beyond a characterization as just another model. A detailed study of its properties is still the focus of current research ${ }^{28}$ and its 
predictions have influenced the interpretation of other numerical and analytic studies. ${ }^{15-17}$ In addition to its physical underpinnings, the BLM is often said to have some mathematical justification for large undercoolings as $\Delta \rightarrow 1^{-}$, where the boundary layer thickness $l$ in Eq. (1.7) is small compared to the radius of curvature of the interface, so that a local model makes good sense. Indeed the BLM reproduces the exact Ivantsov parabola to first order in $(1-\Delta) .{ }^{10}$

For these reasons, we felt it worthwhile to take the ideas behind the BLM seriously, and to see whether it (or some modified version) could be systematically derived from a study of the full heat-conduction equation. A key idea in the BLM was to introduce a physically motivated balance equation for the total heat content $H$ in a tube normal to the interface. Contributing to the dynamics of $H$ is the heat surplus released at the interface, and when $d_{0}$ is nonzero, a "heat-conduction" term describing heat flow between adjacent tubes. This equation, together with some exact geometric relations describing properties of the growing interface, leads directly to the BLM. ${ }^{10}$

We have been able to generalize and justify many of these ideas. ${ }^{18}$ In Sec. IV we introduce a set of timedependent curvilinear coordinates $\left(u_{1}, u_{2}\right)$ that describe properties of the growing interface and the associated temperature field. Different coordinate systems imply different shapes for the tubes normal to the interface and we derive in Sec. $\mathrm{V}$ an exact equation of motion for $H$ that is valid for any (arbitrary) choice of coordinates. Terms in the exact equation involves integrals over all space of the temperature and other associated fields, and have a physically suggestive interpretation. Indeed with one important exception, discussed below, all the terms we find have their counterpart in the BLM. We can reproduce the BLM by choosing a particular coordinate system and approximating some quantities in the integrals by their values at the interface, which seems plausible for $\Delta$ near 1 where the boundary layer near the tip is "thin."

Nevertheless, on closer inspection, the most dramatic and celebrated result of the BLM - the breakup of the Ivantsov family of solutions-turns out to depend on the number of rather specific and arbitrary properties of the model. The reasons for this are described in Secs. V-VII below, and in the remainder of this section, we briefly outline some of these points.

\section{A. Long-time versus short-time behavior}

The BLM successfully reproduces the initial MullinsSekerka instability, ${ }^{10}$ and as such appears to have all the ingredients needed to describe the short-time behavior of a pattern at high undercoolings. Moreover, it gives the right qualitative long-time behavior of highly symmetric shapes like planes, spheres, and cylinders. ${ }^{10}$ However, the steady-state (long-time) growth of a needle crystal is more subtle than these simple cases in that it involves nonlocal effects associated with the $V(\partial T / \partial z)$ term in the steadystate temperature equation in the co-moving frame,

$$
V \frac{\partial T}{\partial z}+D \nabla^{2} T=0
$$

This term breaks the symmetry in the $z$ direction and is crucial for steady-state growth since it determines how the heat released near the tip piles up in the tail region. As a result, a perturbation at the tip has a much larger effect on the tails than a perturbation in the tails would have on the tip. One consequence of this term in the exact boundary-layer formulation discussed in Sec. $\mathrm{V}$ is that the tubes defining $H$ change their shape as they move in time towards the tails of the needle. This gives rise to a "heatconvection" term $J_{\text {conv }}$, which was not included in the BLM. While $J_{\text {conv }}$ vanishes for symmetric solutions like spheres, planes, and cylinders, it is clearly nonzero for needle solutions even in the Ivantsov limit. As we will discuss later, contributions from $J_{\text {conv }}$ are indeed more important for steady-state solutions than the heatconduction effects that dominate the structure of the BLM with $d_{0}>0$.

\section{B. Structure of phase space}

In the steady state, Eqs. (1.1)-(1.4) can be written using Green's function techniques as an integro-differential equation that incorporates the nonlocal spatial and temporal dynamics of heat flow. ${ }^{1}$ The model equations replace this by a set low-order differential equations relating the interface curvature $\kappa$ to the arc length $s$. In the Ivantsov limit, both the BLM and the GM express $\kappa$ as an algebraic function of $\theta$, the angle between the interface normal and the $z$ axis. Since $\kappa=\partial \theta / \partial s$, this relation is a differential equation (in a one-dimensional phase space) that can be solved to determine the shape of the interface. A family of solutions is found. With $d_{0}$ nonzero, the models predict that the dimension of the phase space changes as higher derivatives $\partial \kappa / \partial s$ (present only in the BLM) and $\partial^{2} \kappa / \partial s^{2}$ appear, but all these are multiplied by the small parameter $d_{0}$. It is well known that such terms can lead to singular behavior. ${ }^{29}$ Steady-state solutions of both models can be represented as a flow in a finitedimensional $(\theta, \kappa, \partial \kappa / \partial s)$ phase space. It is found that in the absence of crystalline anisotropy and with $d_{0}$ nonzero, there are no solutions with the proper smooth behavior at the tip ( $\partial \kappa / \partial s=0$ at $\theta=0$ ) that also flow to the physically relevant (Ivantsov) fixed point $\kappa=\partial \kappa / \partial s=0$ representing the tail of the needle at $\theta=\pi / 2$.

It is the very different phase-space structure for $d_{0}$ nonzero in the BLM and the GM that is mainly responsible for the breakup of the Ivantsov family of solutions. However, on expanding the integro-differential equation for the interface temperature, or equivalently, using the exact boundary-layer formation discussed in Secs. IV-VI below, we find that in general there should be derivative terms that survive in the limit $d_{0}=0$. (Since the $J_{\text {conv }}$ term mentioned above is nonzero even for $d_{0}=0$, it is one source for such terms. See Sec. $V$ for details.) On dimensional grounds, an Ivantsov derivative of order $n$ in a local approach has the form

$$
l^{n} \frac{\partial^{n} \kappa l}{\partial s^{n}}
$$

while other non-Ivantsov terms of the same order must be of the form 


$$
d_{0}^{m} l^{n-m} \frac{\partial^{n} \kappa l}{\partial s^{n}}, \quad 1 \leq m \leq n
$$

Here $l$ is the boundary-layer thickness (1.7), which diverges in the tails. Thus, if we expand up to a given order $n$, we expect the Ivantsov-like terms (2.2) always to dominate over the non-Ivantsov-like terms (2.3), since $d_{0} / l<<1$. This suggests that the structure of the flow in phase space in the tails is actually governed by the Ivantsov-like terms absent in the BLM (Ref. 30) and that if a family of perturbed Ivantsov solutions exists, so does a family of $d_{0} \neq 0$ solutions.

\section{Response to perturbations: A thought experiment}

We argued in Sec. II B that the (one-dimensional) phase-space structure of the BLM with $d_{0}=0$ is artificial. Indeed, the absence of derivative terms causes the Ivantsov solutions of the BLM to have an unphysical response to perturbations, as the following thought experiment illustrates.

Let us imagine two materials with different heats of fusion $L_{1}$ and $L_{2}$. Since a difference in the heat of fusion $L$ corresponds to a difference in the dimensionless undercooling $\Delta$, the two Ivantsov solutions corresponding to the cases 1 and 2 and moving at the same velocity $V$ are the two curves (in two dimensions)

$$
z=-\frac{1}{2 p_{1}} x^{2} \text { and } z=-\frac{1}{2 p_{2}} x^{2} .
$$

Here we have written all lengths in units of the diffusion length $2 D / V$, and $p_{1}$ and $p_{2}$ are the dimensionless Péclet numbers [see Eq. (1.6)] corresponding to the undercoolings $\Delta_{1}$ and $\Delta_{2}$, respectively. These two solutions are sketched in Fig. 2(a).

To discuss the response to perturbations in the BLM, we now imagine a fictitious material whose latent heat of fusion $L$ is equal to $L_{1}$ for $|x|<x_{0}$, and then jumps discontinuously to a value $L_{2}\left(<L_{1}\right)$ for $|x|>x_{0}$. For this case, the BLM predicts that there still exists a family of Ivantsov-like solutions, whose shape consists of the superposition of the two curves in 2.4 ,

$$
\begin{aligned}
& z=-\frac{1}{2 p_{1}} x^{2} \text { for }|x|<x_{0}, \\
& z+c_{0}=-\frac{1}{2 p_{2}} x^{2} \text { for }|x|>x_{0},
\end{aligned}
$$

where $c_{0}=\frac{1}{2} x_{0}^{2}\left(1 / p_{1}-1 / p_{2}\right)$. This solution is sketched in Fig. 2(b).

According to the BLM, the two parabolas join without any distortions at $x= \pm x_{0}$ as shown in Fig. 2(b). Physically, however, it is clear that any possible steady-state shape must significantly deviate from parabolic in a finite region around $x_{0}$ of order of the diffusion length because of heat flow in the boundary layer away from the interface-see Fig. 2(c). Since the BLM has no flow terms like $\partial \kappa / \partial s$, etc., that survive for $d_{0}=0$, the shape cannot respond to the perturbation in a finite region about $x_{0}$, as is physically required. A model that had derivative terms even in the Ivantsov limit would eliminate this artificial response. (a)

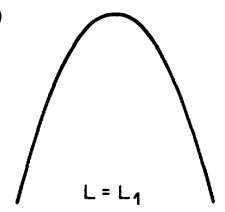

(b)





(c)

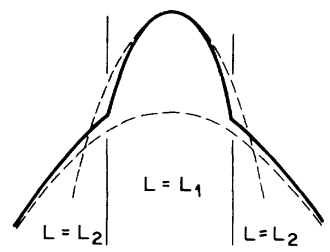

FIG. 2. (a) Steady-state needle solutions moving at the same velocity $V$ for two materials with heat of fusion $L_{1}$ and $L_{2}$ with $L_{1}>L_{2}$. (b) Hypothetical material has latent heat $L=L_{1}$ inside fixed vertical lines and $L_{2}$ outside. According to the BLM, the steady-state solution for $d_{0}=0$ (heavy line) is a superposition of the two curves in (a). (c) In reality, the shape (heavy line) of any possible steady-state solution will deviate from parabolic (dashed lines) near the latent heat discontinuity in a finite region of order the diffusion length.

\section{Arbitrariness of the global flow in phase space}

In a boundary-layer approach, one tries to take advantage of the fact that for $1-\Delta$ small, the steady-state interfaces are gently curved, so that the radius of curvature of the interface is much larger than the boundary-layer thickness $l$. [Recall that a plane with $\kappa=0$ grows for $\Delta \geq 1$.] For $\Delta$ close to 1 , one has $\kappa l=(1-\Delta) f(\theta)$ $+O(1-\Delta)^{2}$. (See Sec. VIII.) Since $\kappa=\partial \theta / \partial s$, we have $l^{2} \partial \kappa / \partial s=l^{2} \kappa \partial \kappa / \partial \theta=O(1-\Delta)^{2}$, etc., and successive derivatives $l^{n+1} \partial^{n} \kappa / \partial s^{n}$ of the smooth steady state solutions order in powers of $(1-\Delta)$. This suggests that one can make a local expansion as $\Delta \rightarrow 1^{-}$and truncate it so that only derivatives of relatively low order appear. In this way, one can indeed formally compute the smooth steady-state profiles in a power series in $(1-\Delta)$, assuming such solutions exist, as shown in Sec. VIII.

Can one also use this expansion to establish the actual existence of these smooth steady-state profiles? It turns out that this is impossible for free dendritic growth. The difficulty is related to a problem already apparent from the inhomogeneous Ivantsov problem of Fig. 2. In the matching region, $x$ near $x_{0}$, the interface curvature changes rapidly over a distance of order of the thermal diffusion length; hence there an expansion assuming slow variations is inconsistent.

In mathematical terms, one can use the ordering property for derivatives of smooth solutions to compute systematically a $(1-\Delta)$ expansion of a smooth solution, assuming it exists. However, the model differential equation generated by the truncation to a finite-dimensional phase space by assuming smoothness does not in general properly describe the behavior of neighboring trajectories 
that can rapidly flow towards, or away from, the smooth solution. Yet it is precisely the global structure of phase space around the smooth solution that determines whether or not a family of such solutions exists in the model equations, as is seen most easily by an application of a "counting argument" for the number of stable and unstable directions at the fixed point in the tails. As a result, predictions about the existence of steady-state solutions given by model equations for dendritic growth derived in this way cannot be trusted.

To illustrate these points, we study a simple model integral equation

$$
p(x)+\epsilon^{-1} \int_{0}^{x} d y e^{-(x-y) / \epsilon} p(y)=e^{-x},
$$

as $\epsilon \rightarrow 0^{+}$. We can obtain the exact solution by differentiation, yielding the differential equation

$$
\epsilon p^{\prime}(x)+2 p(x)=(1-\epsilon) e^{-x}, p(0)=1 .
$$

The solution of $(2.7)$ is

$$
p(x)=\left(\frac{1-\epsilon}{2-\epsilon}\right) e^{-x}+\left(\frac{1}{2-\epsilon}\right) e^{-2 x / \epsilon} .
$$

There is stable "slow" asymptotic behavior going as $e^{-x}$, with a very rapidly decaying transient.

We can also examine the slow solution of (2.6) by a local analysis like that done in the BLM. Expanding $p(y)$ about $p(x)$ in the integral in (2.6), we find, ignoring terms of $O\left(e^{-x / \epsilon}\right)$,

$$
2 p(x)-\epsilon p^{\prime}(x)+\epsilon^{2} p^{\prime \prime}(x)-\cdots=e^{-x} .
$$

There are two ways to analyze (2.9), both of which have their direct counterparts in the exact boundary-layer formulation discussed below in Secs. IV-VII. First, in the spirit of the BLM, let us truncate (2.9) at (say) first order in $\epsilon$ to yield a local model equation

$$
-\epsilon p^{\prime}(x)+2 p(x)=e^{-x} .
$$

The solution to Eq. (2.10) indeed has a slow asymptotic part, which, to first order in $\epsilon$, is

$$
p(x)=\frac{1}{2}\left(1-\frac{\epsilon}{2}\right) e^{-x}
$$

and which agrees with the exact solution (2.8) to first order in $\epsilon$. However, noting that $\epsilon$ is a singular perturbation in (2.10), one might be tempted to conclude that the slow asymptotic behavior is in general unstable, because the solution to (2.10) also has a term of the form $p(x) \sim \exp (2 x / \epsilon)$ that diverges.

The local model (2.10) gives an incorrect assessment of the structural stability of the slow behavior $\epsilon^{-x}$ because the expansion (2.9) is not valid for $p(x)$ (rapidly) varying on the scale $\epsilon^{-1}$. The general phase-space structure of a model derived in this way is quite arbitrary; indeed, the phase-space structure of (2.7) and (2.10) is very different.

As we will see in Secs. IV-VII, the BLM can be obtained by a local expansion essentially identical in spirit to that yielding (2.10). Its predictions, for the stability of slow (smooth) solutions, as well as that of any other model obtained in this way, cannot be relied on, though the form of the smooth solution will be reproduced correctly, as in (2.11).

A consistent analysis of (2.9) can be carried out for the smooth solution, assuming it exists. If we substitute the power-series representation

$$
p(x)=p_{0}(x)+\epsilon p_{1}(x)+\epsilon^{2} p_{2}(x)+\cdots
$$

into (2.9) and solve for the $p_{i}(x)$ recursively, we find

$p_{0}=\frac{1}{2} e^{-x}, \quad p_{1}=-\frac{1}{4} e^{-x}, \quad p_{2}=-\frac{1}{8} e^{-x}, \cdots$.

This reproduces exactly order by order the slow part of (2.8). The analogous expansion for the needle crystal problem is carried out in Sec. VIII. Of course, this method yields only the expansion of the smooth solution, with no information on its stability or existence. However, to try for more from a local analysis is highly problematic, as (2.10) shows.

\section{E. Alternative physical pictures}

Since the various terms included in the BLM have an intuitively clear origin, one might wonder whether, in spite of the above objections, all physically reasonable models could still lead to the same conclusions. We do not believe this to be the case, and will illustrate this by sketching a model with a slightly different but physically motivated heat-flow term that turns out to have completely different behavior. The mathematical reason for the erratic behavior of all such models was discussed in Sec. II D.

As mentioned earlier, a convenient way to obtain a local-interface model is to consider, following Ben-Jacob et al. ${ }^{10}$ an equation for the total heat $H$ contained in some tube perpendicular to the interface (see Fig. 3). By postulating a heat equation for $H$, one then arrives at a particular model. However, the model one gets depends both on the shape of the tube away from the interface and on the approximate heat equation. We now briefly discuss two such choices.

(a) To arrive at the BLM, the tubes have to be chosen in such a way that heat conduction $J_{\text {cond }}$ normal to the tube edges vanishes for $d_{0}=0$; in general it is proportional to (see Sec. VII) $J_{\text {cond }} \approx-D l\left(\partial T_{i} / \partial s\right)$. In order to bring about this simple result, however, the tubes must have ap- (a)

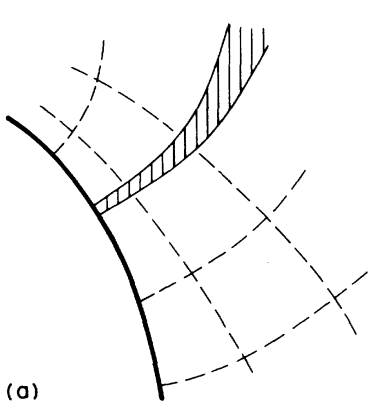

(b)

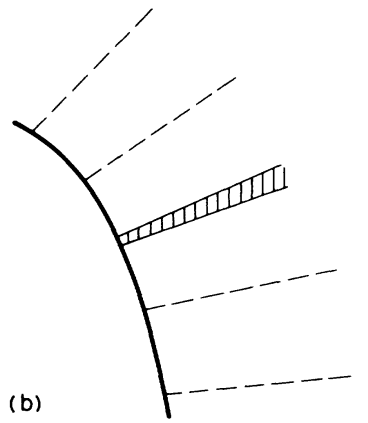

FIG. 3. Different choices of the tubes suggest different model equations. (a) Curved tubes appropriate for the BLM. (b) Another choice (straight tubes) gives heat conduction even in the Ivantsov limit and leads to a different model equation. 
preciable curvature near the interface. To make $J_{\text {cond }}$ vanish in the Ivantsov limit, the tube edges must be normal to the (parabolic) isotherms and follow the shape of the constant $\eta$ lines in the parabolic $(\xi, \eta)$ coordinates of Horvay and Cahn. ${ }^{7}$ See Fig. 3(a). By curving the tubes away from the interface, the heat-conduction term in the BLM by construction becomes very small, and actually vanishes for $d_{0}=0$. Unfortunately a proper accounting of this curvature is difficult to treat in a local picture (which way should the tube bend?) and much of the interesting physics is now hidden in other terms. For example, the convective heat-flow term, $J_{\text {conv }}$ mentioned before in Sec. II A and discussed in detail in Secs. V and VI, is then much larger than $J_{\text {cond }}-$ but $J_{\text {conv }}$ is difficult to guess, and was in fact neglected in the BLM.

(b) Perhaps a more natural way to draw the tubes in a local picture is simply as straight lines normal to the interface, as sketched in Fig. 3(b). In this case, however, one will have heat conduction across the tube edges even when $d_{0}=0$; indeed this is one way to see why in general there should be derivative terms that persist in the Ivantsov limit. Heat conduction with these tubes turns out to have the simple form (see Sec. VII) $J_{\text {cond }} \approx-D(\partial / \partial s)\left(T_{i} l\right)$ $\approx-D(\partial H / \partial s)$, where $H$ is the total heat content per unit length of interface.

If we replace $J_{\text {cond }}$ in the BLM by this expression (still unjustifiably ignoring $J_{\text {conv }}$ ), its behavior changes completely. At large undercoolings the new model for $d_{0}=0$ has a family of steady-state solutions that resemble the needle solutions in the tip [and reproduce the Ivantsov parabola to first order in $(1-\Delta)$ ] but which cross over to flat cigar-shaped behavior in the tails, similar to the behavior in the tails of the GM. ${ }^{9}$ A family of such solutions survives for $d_{0}>0$.

The point of this discussion is not to suggest that this new model is superior to the BLM; in fact the behavior in the tails is physically incorrect and there are other unsatisfactory features. Rather we want to emphasize that underlying the physically pleasing structure of the BLM are implicit and uncontrolled mathematical assumptions that cannot be justified from a strictly local picture, but whose details determine the steady-state predictions of the model.

In the systematic formulation of the boundary-layer approach discussed below in Secs. IV-VIII, the different tubes correspond to choosing different curvilinear coordinate systems away from the interface. We derive an exact equation of motion for $H$ in a tube that is correct for any (arbitrary) choice of curvilinear coordinates, as is physically required. However, different physically motivated approximations to these equations in the spirit of the BLM give completely different results, for the mathematical reasons discussed in Secs. II C and II D.

In Sec. VII we discuss in detail a different model that treats $J_{\text {cond }}$ and $J_{\text {conv }}$ exactly like the BLM, while adding a physically motivated first-derivative term $\partial \kappa / \partial s$ that survives in the Ivantsov limit. The predictions of this new model seem quite satisfactory: for $d_{0}=0$, there is a family of solutions that become parabolic in the tails for all $\Delta$, and also reproduce the exact Ivantsov parabola to first order in $(1-\Delta)$. Contrary to the BLM, however, now a family of solutions is also found for $d_{0}$ nonzero, even though the highest derivative has exactly the same form as in the BLM: $d_{0} \partial^{2} \kappa / \partial s^{2}$. Nevertheless, as argued in Sec. II D, all such predictions are arbitrary and we must look elsewhere for a convincing demonstration of the selection mechanism. We do believe this model is of some mathematical interest for testing the new solvability methods that have been applied to the Hele-Shaw problem ${ }^{12-14}$ and to dendritic growth, ${ }^{15}$ since the existence of the family can be demonstrated by elementary means. It would also be interesting to study the evolution in time of this model and examine possible dynamical implications of its different steady-state structure.

\section{OPEN QUESTIONS CONCERNING OTHER APPROACHES}

Although it is often felt that other numerical and analytical work on dendritic growth has convincingly vindicated the microscopic-solvability scenario, we briefly touch in this section on some open questions we believe still may exist about this issue.

Barbieri, Hong, and Langer ${ }^{15}$ have applied methods developed and successfully employed in the viscous fingering problem ${ }^{12-14}$ to a dendritic growth model in the limit that the Péclet number tends to zero. Although one might think of the Laplace equation as the zero-Pécletnumber limit of the diffusion equation, ${ }^{31,32}$ it is important to remember that the two equations have very different asymptotic properties (cf. the discussion in Sec. I). For dendritic growth (not constrained by walls), the zeroPéclet-number limit is quite singular-the very existence of Ivantsov solutions is a consequence of the physical effects that are responsible for the difference between the two equations. In the approach of Barbieri, Hong, and Langer ${ }^{15}$ perturbations away from the Ivantsov solution for small $d_{0}$ are described by the Laplace equation. They justify it, following Pelcé and Pomeau, ${ }^{33}$ by noting that the integral operator for the difference between the Ivantsov and non-Ivantsov solution has a well-defined limit. However, since neither solution exists for $p=0$ and since the physics of steady-state solutions goes beyond the Laplace equation, it is not clear that this procedure has removed all problems with this very singular limit. We note, e.g., that their final equation appears to have eliminated the asymmetry between response to perturbations in the tip and tail region discussed in Sec. II A, which is a consequence of the $V \partial T / \partial z$ term in Eq. (2.1). It is therefore unclear to us how much their analysis reveals about the full problem with $V$ small but nonzero. However, the consensus of most workers is that much of the structure of the results of Barbieri, Hong, and Langer can be carried over to finite Péclet numbers, so our concerns on this point may well be unfounded. See Sec. $X$ for further discussion.

Numerical work on the full problem by Meiron ${ }^{16}$ and by Kessler et al. ${ }^{17}$ has also been interpreted as consistent with the breakup of a family of solutions. Aside from some technical issues, which will be detailed in future work, ${ }^{19}$ our main reservation with the numerical work 
concerns the method and its interpretation. In both investigations, a method suggested by the local models was used: rather than imposing $z^{\prime}(x=0)=0$ in the program used to compute a two-dimensional symmetric steadystate solution $z(x)[=z(-x)]$, this derivative was left unspecified. For a smooth profile, $z^{\prime}=d z / d x$ should, of course, vanish at $x=0$. In general, then, $z^{\prime}(x=0)$ was found to be small but nonzero; if this value should pass through zero upon varying the velocity at fixed $d_{0}$ (depending on the crystalline anisotropy imposed), this was interpreted as a signal of the existence of a solution at that particular value.

We believe it is difficult to interpret the results of this method. To see this, we have illustrated in Fig. 4 the two scenarios discussed in Sec. I with the analogy of a ball subject to friction $\gamma$ rolling down a hill. The top of the hill corresponds to the tip of the dendrite, and we will think of $\gamma$ as the analogue of the velocity $V$ of a dendrite. In the analogy, the microscopic-solvability picture likens the tail to a local maximum on the right [Fig. 4(a)], so that for arbitrary values of the friction the ball will not roll from the top ("tip"), with initial momentum $P=0$, to the tail.

The $P-x$ phase space corresponding to this situation is sketched in Figs. 5(a) and 5(b). For an arbitrary value of $\gamma$, only one trajectory flows into the tail and there is no trajectory going from the fixed point corresponding to the tip to the tail [Fig. 5(a)]; only for a particular value $\gamma^{*}$ of the friction does such a trajectory exist [Fig. 5(b)]. This corresponds to those special situations in which a steadystate solution is found.

A good way to find numerically the values $\gamma^{*}$ at which a smooth trajectory connecting the two fixed points exists is to integrate the equation in phase space backwards. In general, one will then find $P(x=0) \neq 0$; if $P(x=0)$ changes sign upon varying $\gamma$, this signals the value $\gamma^{*}$ at which a trajectory exists. Note that in this scenario neighboring trajectories diverge near the "tail" fixed point; upon integrating backwards they therefore converge so that the method is numerically stable. The special values of the velocity at which solutions exist in the local models were indeed determined in this way; 9,10 the approach employed to study the full equations is also based on this idea. $^{16,17}$

However the disadvantage is that this approach may well give similar results for other scenarios. In the analogy, our picture views the tail as the attractive fixed point

(a)

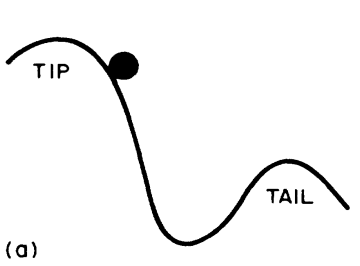

FIG. 4. Particle on hill analogy. (a) Hill structure illustrating microscopic-solvability picture. (b) Hill structure for "attractive tails" as in the contractive flow model, discussed in Sec. VII. of Fig. 5(c) corresponding to the minimum of the potential of Fig. 4. Indeed, for any arbitrary values of $\gamma$ there is now a trajectory with $P=0$ at the tip that flows to the tail. However, this trajectory will in general not be found upon integrating backwards from the tail to the tip as described above, because this approach is now numerically unstable due to the different nature of the fixed point. Instead, one expects to find one of the many well-defined trajectories that have $P(x=0) \neq 0$. As a result, one might erroneously conclude that no "smooth" solution with $P(0)=0$ exists for general $\gamma$.

This description applies to the local models, whose structure differs from the exact integro-differential equations for the interface shape. Hence numerical studies of the full problem do not proceed in quite the way described above; rather they employ Newton's method to determine the whole shape at once. Nevertheless, similar conceptual difficulties appear to exist in that method. If the tails have the properties we argued for physically, it is possible that after relaxing the boundary condition at the tip, both a solution with $d z / d x=0$ and one with $d z / d x \neq 0$ could exist. In the case of multiple solutions, Newton's method might find only the solution with the largest basin of attraction. A similar question of uniqueness of the solutions of the integral equation may also arise in the analytic methods.

These considerations and preliminary numerical work suggest that it might be worthwhile to perform additional tests to interpret the earlier numerical results. Such tests will be reported in a future publication. ${ }^{19}$

\section{SETTING UP A BOUNDARY-LAYER APPROACH}

\section{A. Time-dependent curvilinear coordinates}

We now discuss in detail our generalized boundarylayer approach. In this section we set up a formalism that will allow us to describe the moving interface and the associated temperature field. The analysis is restricted to the case of two dimensions. Let $\mathbf{r}_{i}\left(u_{2}, t\right)$ be the position vector at time $t$ from a space fixed origin to a point on the moving interface, parametrized by the value of some arbitrary coordinate $u_{2}$ expressing displacement along the interface. Intrinsic properties of the curve at that point, such as the curvature $\kappa_{i}$ or arc length $s$ measured along the interface from some fixed line, are independent of the particular choice of coordinate $u_{2}$, which itself could vary in time. A very useful choice for $u_{2}$ when thinking about local properties of models of interface evolution was suggested by Ben-Jacob et al. ${ }^{10}$ and Brower et al. $;^{9} u_{2}$ is chosen so that the velocity $\left(\partial \mathbf{r}_{i} / \partial t\right)_{u_{2}}$ has only a normal component $V_{n}$. Thus derivatives at constant $u_{2}$ correspond to the normal derivatives of Ben-Jacob et al. and Brower et al. If we picture growth as occurring by infinitesimal displacements normal to the interface, then the point with constant $u_{2}$ follows this local motion directly. However, regardless of how the interface actually moves, we can always make such a choice for $u_{2}$ (Brower et al. ${ }^{9}$ term this a "gauge freedom") and it seems most natural for leading to a local description of interface motion.

Here we embed this physically suggestive choice of in- 
(a)

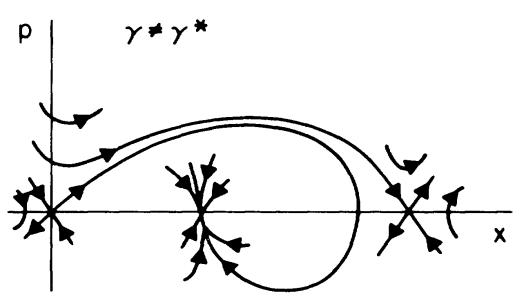

(b)
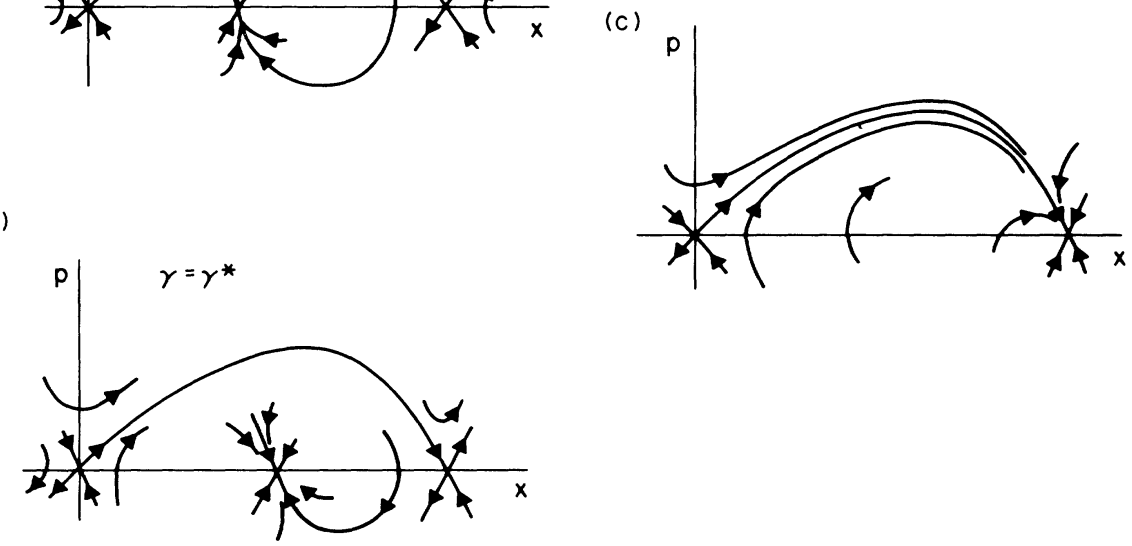

FIG. 5. Phase-space analysis of particle on hill analogy. (a) With fixed hill structure as in Fig. 4(a) and arbitrary friction $\gamma$, the ball cannot roll down the hill with initial momentum $P=0$ and come to rest in the tail. (b) For a particular friction $\gamma^{*}$, the ball rolls from the tip to the tail. (c) With hill structure as in Fig. 4(b), for any $\gamma$, there always is a trajectory connecting tip and tail.

terface coordinates into a general set of time-dependent ${ }^{34}$ curvilinear coordinates $u_{1}, u_{2}$ capable of describing not only the interface but also the temperature field and other properties away from the interface. We let $u_{1}=0$ give the interface position at all times with $u_{1}>0$ indicating points in the liquid phase, while $u_{2}$ at the interface is chosen as indicated above. We require that the coordinates $u_{1}, u_{2}$ away from the interface form an orthogonal system, i.e., the constant $u_{1}$ and $u_{2}$ curves intersecting at each point in space are perpendicular. ${ }^{35}$ A physical displacement $d s_{2}$ along some curve defined by $u_{1}=$ const (referred to hereafter as a $u_{1}$ curve) is given in terms of the scale factor $h_{2}\left(u_{1}, u_{2}, t\right)$ as $d s_{2}=h_{2} d u_{2}$. Similarly for displacement along the orthogonal $u_{2}$ curves we have $d s_{1}=h_{1} d u_{1}{ }^{36}$

Of course, there is a great deal of freedom left in the choice of the coordinates away from the interface. However, physical properties and exact relations are invariant to all such choices. We derive in Appendices $\mathrm{A}-\mathrm{C}$ and in Secs. V and VI below a number of exact relations that hold for any set of orthogonal time-dependent curvilinear coordinates.

Associated with the $u_{1}, u_{2}$ and $h_{1}, h_{2}$ fields are several other fields with useful properties. As shown in Appendix $\mathrm{A}$, the curvatures $\kappa_{j}$ of the $u_{j}$ lines $(j=1,2)$ are given by $\kappa_{1}=\left(h_{1} h_{2}\right)^{-1} \partial h_{2} / \partial u_{1}=\partial \theta / \partial s_{2}$ and $\kappa_{2}=\left(h_{1} h_{2}\right)^{-1} \partial h_{1} / \partial u_{2}=-\partial \theta / \partial s_{1}$. Here $\theta$ is the angle between the (space-fixed) $z$ axis and the unit normal $\hat{\mathbf{a}}_{1}$ to the $u_{1}$ lines (see Fig. 6), and is defined by $\cos \theta=\hat{\mathbf{z}} \cdot \hat{\mathbf{a}}_{1}$. The arc length $s_{2}\left(u_{1}, u_{2}, t\right)$ is measured from the intersection of the $u_{1}$ curve with the $z$ axis, while $s_{1}$ is measured from the interface $u_{1}=0$. When referring to values at the interface $u_{1}=0$, we add a subscript $i$, e.g., $\kappa_{1 i}, \theta_{i}$, and $s_{2 i}$, and when no confusion will result, we will write $\kappa_{1 i} \equiv \kappa$ and $s_{2 i} \equiv s$.
Other fields describe the time-dependence of our coordinates. ${ }^{37}$ As shown in Fig. 6, we introduce velocity fields $V_{1}$ and $V_{2}$ giving the normal velocities in the fixed lab frame of the $u_{1}$ and $u_{2}$ lines. In terms of the position vector $\mathbf{r}$ from some fixed origin in the lab frame, these satisfy

$$
\left(\frac{\partial \mathbf{r}}{\partial t}\right)_{u}=V_{1} \widehat{\mathbf{a}}_{1}+V_{2} \widehat{\mathbf{a}}_{2},
$$

where $\widehat{\mathbf{a}}_{j}$ is the unit normal vector to the lines $u_{j}=$ const. Here the notation ()$_{u}$ indicates a derivative at constant $u_{1}$ and $u_{2}$. At the interface $u_{1}=0$ we have the boundary conditions

$$
\begin{aligned}
& V_{1 i}=V_{n}, \\
& V_{2 i}=0,
\end{aligned}
$$

where $V_{n}$ is the normal interface velocity appearing in Eq. (1.4). Equation (4.3) insures that the interface velocity at fixed $u_{2}$ has only a normal component. However, it is important to note that in general away from the interface we must have $V_{2} \neq 0$, since the behavior of the $V_{2}$ field is coupled to that of the $h_{j}, \kappa_{j}$, and $V_{1}$ fields (see below).

In Appendix A we give a detailed mathematical discussion of our time-dependent curvilinear coordinate system and derive a number of exact geometric relations between the $h_{1}, h_{2}$ and $V_{1}, V_{2}$ fields in terms of the curvatures $\kappa_{1}, \kappa_{2}$. These relations hold for any choice of orthogonal curvilinear coordinates away from the interface.

\section{B. Choice of coordinates away from the interface}

When we wish to consider a specific coordinate system, it is convenient to define it implicitly by requiring that the exact temperature field $T\left(u_{1}, u_{2}, t\right)$ have some particular 


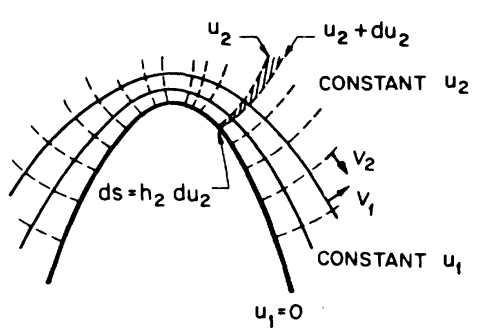

(a)

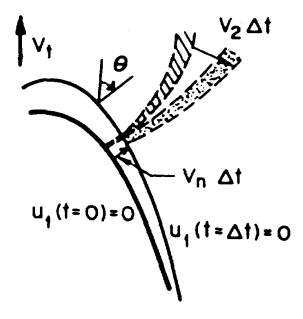

(b)

FIG. 6. Interface and the associated curvilinear coordinate fields $u_{1}$ and $u_{2}$. The interface position at all times is given by $u_{1}=0$ (heavy line). The total heat content in the tube bounded by lines of constant $u_{2}$ and $u_{2}+d u_{2}$ is $H d s ; V_{1}$ and $V_{2}$ are the normal velocities of the constant $u_{1}$ and $u_{2}$ lines. Since $V_{2} \neq 0$ for $u_{1}>0$, the tube deforms as a function of time, giving rise to $J_{\text {conv }}$ as illustrated in (b).

form when expressed in terms of the $u_{1}$ and $u_{2}$ coordinates. In effect, the heat-conduction equation for the temperature field then becomes an equation for the appropriate scale factors $h_{1}\left(u_{1}, u_{2}, t\right)$ and $h_{2}\left(u_{1}, u_{2}, t\right)$ that permit a solution of the desired form.

A particularly useful choice, both for algebraic convenience in carrying out the series expansions discussed in Sec. VIII and because it suggests approximations leading to the BLM equation of Ben-Jacob et al., ${ }^{10}$ arises when we require

$$
T\left(u_{1}, u_{2}, t\right)=T_{i}\left(u_{2}, t\right) e^{-u_{1}} .
$$

The interface temperature $T_{i}$ satisfies the Gibbs-Thomson boundary condition

$$
T_{i}=\Delta-d_{0} \kappa,
$$

where $\kappa\left(=\kappa_{1 i}\right)$ is the interface curvature. (For simplicity we neglect interface kinetics.) Note that when $d_{0}=0$, lines of constant $u_{1}$ in (4.4) are isotherms. This is also the case for the constant $\xi$ curves in the steady-state needle crystal solution of Horvay and Cahn, ${ }^{7}$ which uses parabolic coordinates $(\xi, \eta)$. Thus in this special case there is a particularly simple relationship between the $\left(u_{1}, u_{2}\right)$ coordinates implicitly defined by (4.4) and the parabolic $(\xi, \eta)$ set. In Appendix $D$ we give the equation that results when (4.4) is required to be an exact solution of the temperature equation.

Another choice, equally valid for exact work but suggesting different approximations, is to require

$$
T\left(u_{1}, u_{2}, t\right)=T_{i} e^{-u_{1} / l\left(u_{2}, t\right)},
$$

where the boundary-layer thickness $l\left(u_{2}, t\right)$ is defined in Eq. (1.7). The form of Eq. (4.6) is suggested from the exponential decay on the length scale $l$ of the temperature field in front of a moving plane ${ }^{1}$ with $\Delta=1$. We might hope that a steady-state needle crystal solution for $\Delta$ near 1 could be put into the form (4.6) with a scale factor $h_{1}\left(u_{1}, u_{2}, t\right)$ that does not vary significantly from unity, its value for the moving plane.

The different choices of coordinate system exemplified

by (4.4) or (4.6) show up immediately as different boundary conditions that the $h_{1}$ field must satisfy at the interface $u_{1}=0$. Imposing the heat-conservation condition (1.4) at the interface we have with the definition (1.7) for $l$,

$$
V_{n} \equiv \frac{D T_{i}}{l}=-D(\nabla T)_{n}=-\frac{D}{h_{1 i}} \frac{\partial T}{\partial u_{1}}
$$

Here $h_{1 i}$ is the value of $h_{1}$ at the interface. Thus the coordinate system implied by Eq. (4.4) has

$$
h_{1 i}=l \text {, }
$$

while that generated by (4.6) has

$$
h_{1 i}=1 \text {. }
$$

Perhaps the difference between these coordinate systems is most apparent when one examines the curvature $\kappa_{2 i}$ of the $u_{2}$ lines intersecting the interface. Using Eq. (A4), we see that Eq. (4.8) gives in general a nonzero curvature

$$
\kappa_{2 i}=\frac{1}{l} \frac{\partial l}{\partial s}
$$

to the $u_{2}$ lines, while the alternate coordinate system implied by (4.6) has

$$
\kappa_{2 i}=0 \text {. }
$$

Thus this latter coordinate system is more nearly rectangular (at the interface) than is that given by (4.4), and indeed might seem more "natural" in a local picture of interface evolution. Figure 3 shows qualitatively these two choices for an Ivantsov needle. However, the coordinate system generated by (4.4) turns out to be the one that naturally leads to the BLM equation, and it is also easier to use in most algebraic work because of its simple form in the Ivantsov limit. Hence we use it for most of the rest of this paper when a specific choice is required, and return to the alternate (4.6) only in Sec. VII where model equations are discussed. Most results, including the diffusion of the heat content in the boundary layer given in Sec. V, hold whatever choice we make.

\section{BALANCE EQUATION FOR HEAT CONTENT IN THE BOUNDARY LAYER}

As pointed out by Ben-Jacob et al., ${ }^{10}$ it useful to examine the behavior in time of $H$, the total heat content per unit arc length of the interface. In this section we derive an exact result for $(\partial H / \partial t)_{u_{2}}$, which is easily related to approximations introduced by Ben-Jacob et al. We define $H$ as

$$
\begin{aligned}
H\left(u_{2}, t\right) \equiv \frac{1}{h_{2 i}} \int_{0}^{\infty} & d u_{1} h_{1}\left(u_{1}, u_{2}, t\right) \\
& \times h_{2}\left(u_{1}, u_{2}, t\right) T\left(u_{1}, u_{2}, t\right),
\end{aligned}
$$

where $h_{2 i}=h_{2}\left(u_{1}=0, u_{2}, t\right)$. Note that $H d s_{2 i} \equiv H h_{2 i} d u_{2}$ is the total heat content in the tube between lines of constant $u_{2}$ and $u_{2}+d u_{2}$, as illustrated in Fig. 6, and that integrating $H d s_{2 i}$ over all tubes along the interface indeed 
gives the total heat content.

It is clear physically that three effects can contribute to the change in time of the heat content in a tube: (i) the "heat surplus" generated at the interface, (ii) heat diffusion across the constant- $u_{2}$ lines, and (iii) the motion of the tubes with respect to the lab frame. This effect is illustrated in Fig. 6(b); it can be seen that the tube boundaries will in general move in time because of deformations in shape (e.g., the stretching sketched in the figure) as well as due to the normal motion of the interface. (Even if a curved tube does not deform, displacement of the tube normal to the interface still causes motion of the tube boundaries away from the interface.) In the lab frame, the tube boundaries thus move with a velocity $V_{2}$ normal to themselves, with $V_{2}=0$ at the interface. Alternatively, when following a tube, points in the lab frame move with a velocity $-V_{2}$ across the tube boundaries, and the resulting term in the heat-balance equation can be viewed as a "convective" heat flow.

In Appendix B we calculate $(\partial H / \partial t)_{u_{2}}$ using (5.1) and the kinematic equations in Appendix $\mathrm{A}$ and find the exact result

$$
\begin{aligned}
(\partial H / \partial t)_{u_{2}}= & V_{n}\left(1-T_{i}\right)-\partial J_{\text {cond }} / \partial s \\
& -\partial J_{\text {conv }} / \partial s-H V_{n} \kappa
\end{aligned}
$$

where

$$
\begin{aligned}
& J_{\text {cond }} \equiv-D \int_{0}^{\infty} d u_{1}\left(\frac{h_{1}}{h_{2}}\right) \frac{\partial T}{\partial u_{2}}=-D \int_{0}^{\infty} d s_{1} \frac{\partial T}{\partial s_{2}} \\
& J_{\text {conv }} \equiv-\int_{0}^{\infty} d u_{1} h_{1} V_{2} T=-\int_{0}^{\infty} d s_{1} V_{2} T
\end{aligned}
$$

Again these equations hold for any choice of coordinate system away from the interface.

The first three terms on the right-hand of (5.2) are the contributions (i)-(iii) discussed above. The fourth term takes account of the fact that the interface arc length $d s_{2 i}$ of the constant- $u_{2}$ tube increases during growth of a positively curved interface since constant- $u_{2}$ points grow apart in time. Thus $H$, the heat content per unit length of interface, decreases. Equation (5.2) is one of the basic results of this paper.

All the terms in (5.2) except the $J_{\text {conv }}$ term had been anticipated in the physical arguments that lead Ben-Jacob et $a l .{ }^{10}$ to the BLM equation. Indeed, as we show below, simple approximations to (5.2) yield directly the BLM. Furthermore, the $J_{\text {conv }}$ term omitted in the BLM plays no essential role in the Mullins-Sekerka instability and it vanishes identically by symmetry for a growing plane or sphere, all these being cases where the BLM was found to capture the crucial physics. ${ }^{10}$ However, as we now show, for the steady-state needle crystal problem there is an intimate connection between heat conduction expressed in $J_{\text {cond }}$ and "heat convection" given by $J_{\text {conv }}$, which depends on the (arbitrary) choice of coordinates away from the interface, and both terms must be considered. Unfortunately, even when this is done, one still cannot rely on the steady-state predictions of the resulting model equations because of the general objections discussed in Sec.
II D and amplified below.

\section{EXACT STEADY-STATE EQUATIONS}

In the steady state we follow Ben-Jacob et al. and reexpress the time derivative $(\partial H / \partial t)_{u_{2}}$ in terms of a spatial derivative, using the steady-state chain rule given in $\mathrm{Eq}$. (C7). Appendix C discusses in some detail the steadystate simplifications of the results of Appendix A. In particular, the normal interface velocity satisfies $V_{n}$ $=V \cos \theta_{i}$. Thus (5.2) becomes, on using (4.2), (4.3), and (C7),

$$
\begin{aligned}
& \left(\frac{\partial H}{\partial s}\right) V \sin \theta_{i}+H V \cos \theta_{i} \kappa \\
& =\left(1-T_{i}\right) V \cos \theta_{i}-\frac{\partial}{\partial s} J_{\text {conv }}-\frac{\partial}{\partial s} J_{\text {cond }},
\end{aligned}
$$

or, since $\kappa=\partial \theta_{i} / \partial s$,

$$
\frac{\partial}{\partial s}\left(H \sin \theta_{i}+\widetilde{J}_{\text {conv }}+\widetilde{J}_{\text {cond }}\right)=\left(1-T_{i}\right) \cos \theta_{i},
$$

where $\widetilde{J} \equiv J / V$. Equation (6.2) is exact, holds for any choice of coordinates away from the interface, and is the starting point for all of our analysis in the steady state. ${ }^{38}$

As shown in Appendix C, Eq. (C9), we can also rewrite (6.2) exactly in terms of the simpler but less physically transparent quantity

$$
M \equiv \int_{0}^{\infty} d u_{1} h_{1} \sin \theta T,
$$

as

$$
\frac{\partial}{\partial s}\left(M+\widetilde{J}_{\text {cond }}\right)=\left(1-T_{i}\right) \cos \theta_{i}
$$

Thus the effect of the $\widetilde{J}_{\text {conv }}$ term in (6.2) is implicitly and exactly taken into account if we consider $M$ rather than $H \sin \theta_{i}$. This simplification holds only in the steady state. The compact form of Eq. (6.4) is useful in the algebraic manipulations leading to the $(1-\Delta)$ expansion given in Sec. VIII, but for the discussion of model equations, it seems more profitable to use (6.2), and deal with the same quantities $H, J_{\text {conv }}$, and $J_{\text {cond }}$, that also occur in the timedependent problem (5.2).

There are at least two ways we can exploit (6.2) or (6.4). The first is to use it to generate an expansion of those physically relevant steady-state solutions that join smoothly onto the Ivantsov parabola far from the tip. As discussed in Sec. II D, successive derivatives of such smooth solutions can be ordered in powers of $(1-\Delta)$. This expansion is carried out in Sec. VIII and is directly analogous to the expansion (2.12) of the solution to the model equation (2.6) discussed in Sec. II D. However, this method gives no information about whether there exists a continuous family, a discrete set, or even no such solutions with the assumed smoothness properties. Furthermore the $(1-\Delta)$ expansion is asymptotic (even for the Ivantsov solution) and so could miss some important features of the true smooth steady-state solutions, assuming they do exist. Thus, the results of the $(1-\Delta)$ expansion have at best only a qualitative validity. 
The second way to exploit (6.2) leads directly to the model equations, but the justification is much less apparent. The idea is to try to express $H, J_{\text {conv }}$, and $J_{\text {cond }}$ in terms of $\theta_{i}, \kappa\left(=\partial \theta_{i} / \partial s\right), \partial \kappa / \partial s, \ldots$ If this is possible, then (6.2) becomes a differential equation for $\kappa$ as a function of $\theta_{i}$, which could in principle be solved to obtain the steady-state profile. Further, one could hope to use the differential equation to determine whether the physically relevant smooth solutions actually exist. This is the basic idea of the BLM of Ben-Jacob et al. ${ }^{10}$

Of course, determining the functional dependence $H\left(\theta_{i}, \kappa l, l^{2} \partial \kappa / \partial s, \ldots\right)$, etc., is very difficult. Indeed from (5.1), (5.3), and (5.4) we see that $H, J_{\text {conv }}$ and $J_{\text {cond }}$ involve integrals over all space. Thus a representation in terms of the interface values of $\kappa$ and its derivatives must, formally speaking at least, involve derivatives of arbitrarily high order (generated, say, from terms in the Taylor expansions of the $h_{1}, h_{2}$, and $V_{2}$ fields about $u_{1}=0$ ). Further, since we wish to determine the conditions under which smooth physical solutions exist, we can use no smoothness assumption to eliminate the higher-order derivatives $\partial^{n} \kappa / \partial s^{n}$. Indeed, since the driving force is large near $\Delta=1$ we expect on physical grounds (see Sec. II C) that an arbitrary trajectory could vary appreciably on the short length scale $l$.

An analysis of the general problem thus must take account of possibly singular solutions with $\kappa$ varying rapidly with respect to $s$, so that $l^{2} \partial \kappa / \partial s=O(\kappa l)$, etc. A proper description of such solutions requires an essentially infinite-dimensional phase $\operatorname{space}^{39}\left(\theta, \kappa l, l^{2} \partial \kappa / \partial s, \ldots\right)$. An arbitrary truncation to (say) a three-dimensional phase space as in the BLM cannot be relied on to reproduce the proper stability, or lack thereof, of the smooth solution. This was illustrated in Sec. II D for the local approximation (2.10) to the integral equation (2.6). The higher-order derivative terms $l^{n+1} \partial^{n} \kappa / \partial s^{n}, n>3$, while small [of the order of $O(1-\Delta)^{n}$ ] for smooth solutions, play an important role in describing the behavior of an arbitrary trajectory where $l^{2} \partial \kappa / \partial s=O(\kappa l)$, etc., and hence in determining if a smooth solution actually exists. As we will now see, this leads to great variability in the predictions of different model equations obtained by such truncations.

\section{MODEL EQUATIONS}

To obtain model equations from Eq. (6.2), we pick a particular coordinate system away from the interface and approximate $h_{1}, h_{2}$, and $V_{2}$ in the integrals defining $H$, $\widetilde{J}_{\text {conv }}$, and $\widetilde{J}_{\text {cond }}$ in terms of their values at the interface. Although (6.2) is exact and holds for any choice of coordinate system, different choices of coordinates away from the interface turn out to suggest different models, with very different properties. Similarly for a given coordinate system, we can obtain different results depending on how we approximate $h_{1}, h_{2}$, and $V_{2}$ in Eq. (6.2). This, of course, suggests that the results of any model we obtain in this way should be viewed with considerable skepticism.

Since we have argued that there is no mathematically justified procedure for deriving model equations, we will first consider some physically motivated approximations in the spirit of the BLM and briefly discuss the models that result. Mathematical details are given in Appendix E. The first truncation leads immediately to the BLM equation of Ben-Jacob et al. ${ }^{10}$ Let us use the coordinate system generated by (4.4) and approximate $h_{1}, h_{2}$, and $V_{2}$ by their values at the interface in the integrals (5.1), (5.2), and (5.4). This may seem very plausible for $\Delta \rightarrow 1^{-}$where there is a thin boundary layer. This approximation represents the first term in the Taylor-series expansion of these quantities about their interface values and is the starting point for the systematic expansion in Sec. VIII. We immediately obtain the approximations used by BenJacob et al. ${ }^{10}$

$$
\begin{aligned}
& H=T_{i} l, \\
& \widetilde{J}_{\text {cond }}=-(D / V) l \partial T_{i} / \partial s=(D / V) l d_{0} \partial \kappa / \partial s, \\
& \widetilde{J}_{\text {conv }}=0,
\end{aligned}
$$

and Eq. (5.2) reduces to the time-dependent BLM equation studied by Ben-Jacob et al. Substituting (7.1) into (6.2) yields the steady-state BLM equation. ${ }^{40}$ It is most conveniently written as a set of three coupled equations,

$$
\begin{aligned}
& \partial \theta_{i} / \partial s \equiv \kappa, \\
& \partial T_{i} / \partial s=\lambda \text {, } \\
& \partial \lambda / \partial s=\frac{2 V \sin \theta_{i}}{D} \lambda+T_{i} \frac{V \kappa}{D \cos \theta_{i}}-\frac{\lambda^{2}}{T_{i}} \\
& -\left[\frac{V}{D}\right)^{2} \frac{\cos ^{2} \theta_{i}\left(1-T_{i}\right)}{T_{i}}-\kappa \tan \theta_{i} \lambda,
\end{aligned}
$$

where for $d_{0} \neq 0$ we have $\kappa=\left(\Delta-T_{i}\right) / d_{0}$ from Eq. (4.5). For $d_{0}=0$, we have $T_{i}=\Delta, \lambda=0$, and (7.2) reduces to

$$
\frac{D \kappa}{V}=\frac{(1-\Delta)}{\Delta^{2}} \cos ^{3} \theta_{i} \text {. }
$$

This is an equation for a family of parabolas; to first order in $(1-\Delta)$, Eq. (7.3) agrees with the exact Ivantsov expression (1.6) relating the tip curvature to the undercooling. We see directly from the Ivantsov solution that $\kappa l=(1-\Delta) f\left(\theta_{i}\right)+O(1-\Delta)^{2}$, and the same must hold true for any physical smooth solution (7.2) might possess when $d_{0} \neq 0$.

Note from (5.3) that with this choice of coordinate system, $\widetilde{J}_{\text {cond }}$ vanishes identically in the Ivantsov limit, in agreement with the BLM approximation (7.1), and that $\widetilde{J}_{\text {cond }}$ is generally of $O\left((1-\Delta)^{2}\right)$ for smooth solutions. [From (7.1), it follows that $\widetilde{J}_{\text {cond }}=(D / V)\left(d_{0} /\right.$ l) $l^{2} \kappa(\partial \kappa / \partial \theta)=O\left((\kappa l)^{2}\right)=O\left((1-\Delta)^{2}\right)$.] On the other hand, the $\widetilde{J}_{\text {conv }}$ term set equal to zero in the BLM is actually $O(1-\Delta)$ for smooth solutions (it is nonzero even in the Ivantsov limit). Indeed, from (5.4) we get to lowest order $J_{\text {conv }}=-T_{i} l \partial V_{2} / \partial u_{1}$, and according to Eq. (A14) evaluated at the interface, we have $\partial V_{2} / \partial u_{1}=2 D \tan \theta \kappa+\cdots$. Hence $J_{\text {conv }} \approx-2 D \kappa l \tan \theta$ $=O(1-\Delta)$, and the $\widetilde{J}_{\text {conv }}$ term is much larger than the $\widetilde{J}_{\text {cond }}$ term kept in the BLM equation. Thus the BLM with $d_{0}>0$ is never exact, even in the limit $\Delta \rightarrow 1^{-}$. 
We have already discussed the main prediction of the BLM: the nonexistence of smooth steady-state solutions to Eq. (7.2) for $d_{0}>0$ in the absence of anisotropy. ${ }^{10}$ This result is rederived in the more general mathematical treatment of local models given in Appendix E. In Sec. II C we argued that the absence of derivative terms in the BLM equation (7.3) for $d_{0}=0$ is a crucial element in this predictions, and one that can be questioned on both physical and mathematical grounds. The following model extends the phase space in the Ivantsov limit and indeed turns out to give very different predictions.

Let us examine the BLM approximation to $H=T_{i} l$. This is the dominant term and is valid for a plane, but it is clear physically that for fixed boundary-layer thickness $l$, the heat content per unit length of interface is larger for a positively curved interface than for a plane, since the normals to the interface diverge apart. Indeed, this effect underlies the Mullins-Sekerka instability. ${ }^{22}$ Thus we expect on very general grounds an expression for $H$ of the form

$$
H=T_{i} l(1+a \kappa l+\cdots),
$$

where $a$ is some positive coefficient of order unity. [For $d_{0}=0$, the exact Ivantsov solution ${ }^{6,7}$ can be put into the form (7.4) with $a=2$.] We can obtain this $\kappa l$ correction term mathematically by taking the next term in the Taylor-series expansion of $h_{1} h_{2}$ about $u_{1}=0$ in the definition of $H$, Eq. (3.1), noting that $\kappa \equiv \kappa_{1 i}$ $=\left(h_{1} h_{2}\right)^{-1}\left(\partial h_{2} / \partial u_{1}\right)_{i}$ and that according to Eq. (D10) $\partial \ln h_{1} / \partial u_{1}=\kappa l-(1-\Delta)$; this gives Eq. (7.4) with $a=2$. Of course, as argued before we have no real argument for stopping this expansion at first order. Thus we can give no systematic mathematical justification and we suggest strictly as a new physically motivated model equation the use of (7.4) for $H$ with $J_{\text {conv }}$ and $J_{\text {cond }}$ as given by the BLM. For reasons that will become immediately apparent, we call the resulting model the contractive flow model (CFM).

The CFM is analyzed in Appendix E. For $d_{0}=0$ there is a family of solutions that agree with the exact Ivantsov solutions to $O(1-\Delta)$; there are nonparabolic corrections but these are higher order in $(1-\Delta)$. In the tails the shape becomes parabolic for all $\Delta$. The Ivantsov-like needle solution for $\Delta=0.95$ and $a=2$ is represented in Fig. 7 by the solid line and other trajectories in the twodimensional $(\theta, \kappa)$ phase space by the dashed lines. Note that the fixed point at $\theta=\pi / 2$ is stable and all trajectories flow towards it. There is indeed "contractive flow" on following trajectories from the tip region to the tails.

This can perhaps be seen more dramatically for $d_{0}>0$. The family of solutions persists-indeed numerical calculations and analytical considerations agree that there is now a "family of families," where needlelike solutions with a range of $\kappa_{t}$ but satisfying $\kappa_{t}^{\prime}=0$ are found. Figure 8 shows how trajectories that initially lie within the square box for $\theta=0$ are confined to smaller and smaller rectangles as $\theta$ increases, with all trajectories flowing to the Ivantsov fixed point in the tail.

While we doubt that this prediction of "superstable tails" will turn out to be correct for the full problem, the model does show that it is a mathematical possibility, and



FIG. 7. Trajectories in CFM in the Ivantsov limit. Only one trajectory connects the tip with $\partial \kappa / \partial \theta=0$ to the tail at $\theta=\pi / 2$ but all trajectories flow into the tail.

that adding a higher-derivative term can actually increase rather than decrease the number of solutions. ${ }^{41}$ It also illustrates how the predictions of all such models depend on details of the phase-space structure.

Yet another model with different properties arises if we return to the basic BLM approximation of taking only the interface values of $h_{1}, h_{2}$, and $V_{2}$ in (3.1), (3.3), and (3.4) but consider a different coordinate system away from the interface. Clearly this choice can have no effect on any physical property, and indeed the exact expansion in powers of $(1-\Delta)$ discussed in Sec. VIII is unchanged. However, let us choose the coordinate system implied by Eq. (2.6) and evaluate $H, J_{\text {conv }}$, and $J_{\text {cond }}$ by approximating $h_{1}, h_{2}$, and $V_{2}$ by their values at the interface. We find $H=T_{i} l$ and $\widetilde{J}_{\text {conv }}=0$ just as in the BLM but now $J_{\text {cond }}=-D(\partial / \partial s)\left(T_{i} l\right)=-D(\partial H / \partial s)$. With this model we have heat conduction even in the Ivantsov limit and here we again find a two-dimensional phase space. The properties of this model can be analyzed by the methods

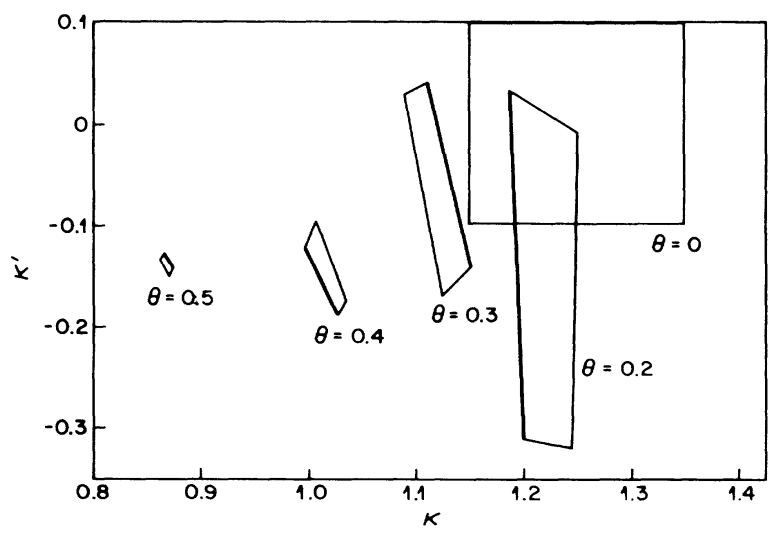

FIG. 8. Contractive flow for $d_{0}>0$ illustrated. Poincaré sections with successive $\kappa, d \kappa / d \theta$ planes are shown for trajectories starting in the initial square at $\theta=0$. The shrinking of the area with increasing $\theta$ illustrates the contraction of the flow, while the side drawn with a heavy line illustrates its rotation. Eventually, all trajectories flow into the fixed point at $\theta=\pi / 2$. 
of Appendix E, and we have also performed some numerical calculation. Some of the results were briefly mentioned in Sec. II E, and we will not discuss them further here.

Finally we analyze in Appendix $\mathrm{E}$ a model with a three-dimensional $(\theta, \kappa, \partial \kappa / \partial s)$ phase space in the Ivantsov limit, obtained by keeping terms up to $O\left((1-\Delta)^{2}\right)$ for smooth solutions in the exact $(1-\Delta)$ expansion for $M$ in Eq. (6.4), as described in detail in Sec. VIII. The phase space for this model has two stable and one unstable directions in the tail at $\theta=\pi / 2$, consistent with the existence of a family of solutions, while not yielding the superstability of the tails as does the CFM. Thus we have a host of models with different stability properties in the tails, and generating different predictions about the existence of a family of solutions for $d_{0}>0$. We conclude that none of these predictions can be relied on, for the mathematical reasons discussed in Sec. II D.

\section{VIII. $(1-\Delta)$ EXPANSION OF SMOOTH SOLUTIONS}

Consistent results, that do not depend on choice of coordinate system away from the interface, can be obtained if we carry out an expansion in powers of $(1-\Delta)$ of the smooth solutions of (6.2) or (6.4), assuming that such solutions exist. For this purpose it is simplest to use (6.4). Our basic strategy is to expand $h_{1}, h_{2}$, and $\sin \theta$ in a Taylor series about $u_{1}=0$ and thus evaluate the integrals in (6.3) or (5.3) and (5.4). We make use of the ordering property for smooth solutions $l^{n+1} \partial^{n} \kappa / \partial s^{n}=O((1$ $-\Delta)^{n+1}$ ) to help determine the order in $(1-\Delta)$ of the resulting terms. For algebraic simplicity we use the coordinate system (4.4), but it is easy to verify that the different coordinate system (4.6) gives the same results.

The expansion begins by noting that to $O(1-\Delta)$ we can neglect $\widetilde{J}_{\text {cond }}$ [it is $O\left((1-\Delta)^{2}\right)$ for smooth solutions] and replace $h_{1}$ and $\sin \theta$ in (6.3) by their values at the interface. This yields $M \approx T_{i} l \sin \theta_{i} \approx(D / V) \tan \theta_{i}$, since $T_{i}=\Delta-d_{0} \kappa=1+O(1-\Delta)$. [For estimating order in $(1-\Delta)$, it is safe to treat $d_{0}$ as $O(l)$, although in fact $d_{0}<<l$ in all physical applications.] Equation (6.4) then yields $(D / V) \kappa / \cos ^{2} \theta_{i}=\left(1-\Delta+d_{0} \kappa\right) \cos \theta_{i}$, or

$$
\frac{D \kappa}{V}=\frac{(1-\Delta) \cos ^{3} \theta_{i}}{1-d_{0} \cos ^{3} \theta_{i}}+O\left((1-\Delta)^{2}\right),
$$

where $\widetilde{d}_{0} \equiv d_{0} V / D$. The next correction term from the Taylor series involves $\partial \sin \theta / \partial u_{1} \quad\left(=\cos \theta h_{1} \kappa_{2}\right)$ and $\partial h_{1} / \partial u_{1}$ evaluated at the interface. These can be obtained from results shown in Appendix D, Eqs. (D6) and (D11). Equation (D6) holds in general and hence it can be differentiated again with respect to $u_{1}$ to yield higher derivatives in the Taylor series. Its structure is such that a derivative $\partial^{n} / \partial u_{1}^{n}$ generates at most a term of the form $\partial / \partial u_{2}\left(\partial^{n-1} / \partial u_{1}^{n-1}\right)$ and hence it can be evaluated at the interface in terms of the lower-order quantities already calculated. We anticipate, and find for smooth solutions, that each differentiation generates terms of successively higher order in powers of $(1-\Delta)$. See Appendix D for more details.

In this way we can calculate as many terms as we desire of the $(1-\Delta)$ expansion. We have verified that this procedure reproduces the exact Ivantsov solution through $O\left((1-\Delta)^{3}\right)$. Similarly we find for $d_{0} \neq 0$ to $O\left((1-\Delta)^{2}\right)$

$$
\begin{aligned}
& \frac{D \kappa}{V}=\frac{(1-\Delta) C^{3}}{\left(1-\widetilde{d}_{0} C^{3}\right)} \\
& +(1-\Delta)^{2} \frac{3 C^{3}}{\left(1-\widetilde{d}_{0} C^{3}\right)^{4}}\left[1-\widetilde{d}_{0} C^{3}\left(4-3 C^{2}\right)\right. \\
& \left.+\widetilde{d}_{0}^{2} C^{6}\left(1-4 C^{2}+3 C^{4}\right)\right] .
\end{aligned}
$$

Here $C \equiv \cos \theta_{i}$. This equation is consistent with the existence of a family of solutions, parametrized, e.g., by $V$. Of course, we cannot conclude that such a family actually exists since we have assumed the smoothness property in the derivation of (8.2).

\section{TEMPERATURE EQUATION TO $O(1-\Delta)$}

As Sec. VII demonstrated, the possible existence of solutions for which the derivatives of $\kappa$ do not order makes the analysis of truncated differential equations derived from an integrated quantity such as $H$ inconclusive. We therefore return to an analysis of the full steady-state temperature equation, and the only simplification left to us is to assume that $\Delta$ is close enough to 1 that terms of $O\left((1-\Delta)^{2}\right)$ can be ignored. However, we assume no smoothness properties for a general trajectory and take $\kappa$ and its derivatives as being of the same order in $(1-\Delta)$. We use the coordinate system implied by Eq. (4.4).

We assume that we are close enough to the Ivantsov solution that we can linearize around it and write

$$
\kappa_{1}=\kappa_{1}^{\mathrm{Iv}}+\frac{1}{l} \delta \kappa_{1},
$$

with similar definitions for perturbation of $h_{j}^{\mathrm{Iv}}, \kappa_{2}^{\mathrm{Iv}}$, etc. Here the superscript Iv refers to the Ivantsov solution. We choose the prefactor $l$ in (9.1) and related definitions so the perturbations are dimensionless, and derive relations linear in the perturbations and valid to $O(1-\Delta)$. These relations come from the exact kinematic equations discussed in Appendixes $\mathrm{A}$ and $\mathrm{C}$ and the full temperature equation in Appendix D, where we use the coordinate system implied by Eq. (4.4).

In particular, if we consider variations of (D8), equivalent to the full temperature equation, we find

$$
\begin{aligned}
\frac{\partial \delta h_{1}}{\partial u_{1}}-\delta \kappa_{1}-\delta h_{1}+\tan \theta_{i} \delta \theta= & d_{0}\left(-\frac{\partial \delta \kappa}{\partial s} \tan \theta_{i}+l \frac{\partial^{2} \delta \kappa}{\partial s^{2}}\right) \\
& +O\left((1-\Delta)^{2}\right)
\end{aligned}
$$

Note that the non-Ivantsov terms enter as a constant boundary term in (9.2). We shall also need variations of Eq. (A5), which can be written

$$
\frac{\partial \delta \kappa_{1}}{\partial u_{1}}=-l \frac{\partial \delta \kappa_{2}}{\partial s_{2}}+O\left((1-\Delta)^{2}\right) .
$$


These equations must be satisfied subject to the boundary conditions $h_{1 i}=l$ or

$$
\delta h_{1 i}=\tan \theta_{i} \delta \theta_{i}-\left(d_{0} / l\right) \delta \kappa,
$$

or, taking the derivative with respect to $s$,

$$
\delta \kappa_{2 i}=\delta \kappa \tan \theta_{i}-d_{0} \frac{\partial \delta \kappa}{\partial s},
$$

where $\delta \kappa \equiv \delta \kappa_{1 i}$ and $\delta \kappa_{2}=l\left(\partial \delta h_{1} / \partial s_{2}\right)+O\left((1-\Delta)^{2}\right)$.

We anticipate from the form of the equations that solutions involve exponentials and can be written in a product form $f\left(u_{1}\right) g\left(u_{2}\right)$. In particular, we will verify that approximate solutions can be found whose $u_{2}$ dependence for all $\delta h_{j}$ and $\delta \kappa_{j}$ can be written in the convenient form

$$
e^{-A s_{2} / l}
$$

where $A\left(\theta_{i}\right)$ is a smooth function of $\theta_{i}$, the sign of whose real part determines whether perturbations approach the Ivantsov solution in the tails as $s_{2} \rightarrow \infty$. For simplicity we restrict the analysis to $A$ real. Note that a factor like (9.6) in $\delta \kappa$ produces precisely the behavior discussed in Sec. VI where $l \partial \delta \kappa / \partial s_{2}=\boldsymbol{O}(\delta \kappa)$, etc., so that derivatives of $\delta \kappa$ do not order.

Since the right side of (9.2) is independent of $u_{1}$, it is convenient to first differentiate (9.2) with respect to $u_{1}$ and find

$$
\frac{\partial^{2} \delta h_{1}}{\partial u_{1}^{2}}-\frac{\partial \delta \kappa_{1}}{\partial u_{1}}-\frac{\partial \delta h_{1}}{\partial u_{1}}-\tan \theta_{i} \delta \kappa_{2}=0,
$$

where we note $\delta \kappa_{2}=-\partial \delta \theta / \partial u_{1}+O\left((1-\Delta)^{2}\right)$. Further using (9.6) we have $\delta \kappa_{2}=l\left(\partial \delta h_{1} / \partial s_{2}\right)=-A \delta h_{1}$ $+O\left((1-\Delta)^{2}\right)$. Thus (9.3) can be written

$$
\frac{\partial \delta \kappa_{1}}{\partial u_{1}}=-A^{2} \delta h_{1} \text {. }
$$

Finally, then we have in (9.7)

$$
\frac{\partial^{2} \delta h_{1}}{\partial u_{1}^{2}}-\frac{\partial \delta h_{1}}{\partial u_{1}}+\left(A^{2}+A \tan \theta_{i}\right) \delta h_{1}=0 \text {. }
$$

Recalling (9.6), the solution to (9.9) can be written in the form

$$
\delta h_{1}=-e^{-(A / l) s_{2}}\left(c \lambda_{+} e^{\lambda_{+} u_{1}}+\lambda_{-} e^{\lambda_{-} u_{1}}\right) .
$$

One integration constant determines the arbitrary overall normalization in (9.10) and $c$ is the other constant. Here $\lambda_{ \pm}$satisfy the equation derived from (9.9) by assuming a trial solution $e^{\lambda u_{1}}$,

$$
\lambda^{2}-\lambda+\left(A^{2}+A \tan \theta_{i}\right)=0,
$$

and are given by

$$
\lambda_{ \pm}=\frac{1}{2}\left\{1 \pm\left[1-4\left(A^{2}+A \tan \theta_{i}\right)\right]^{1 / 2}\right\} .
$$

The particular form (9.10) allows the solution of (9.8) to be written as

$$
\delta \kappa_{1}=A^{2} e^{-(A / l) s_{2}}\left(c e^{\lambda_{+} u_{1}}+e^{\lambda_{-} u_{1}}+b\right),
$$

where $b$ is another integration constant.

Equation (9.12) shows that $\operatorname{Re} \lambda_{ \pm}>1$ if $-\tan \theta_{i}<A<0$.
This corresponds to a perturbation that blows up on approaching the tails. Since the temperature field in (4.4) goes as $e^{-u_{1}}, \delta h_{1}$ in (9.10) then increases so fast that the heat content in the boundary layer becomes infinite. It thus seems reasonable to exclude these types of perturbations.

On the other hand, all perturbations with $A>0$ (which decay towards the tail) behave in a consistent and physically reasonable fashion, while the only allowed solutions with $A$ negative (i.e., increasing towards the tail) are those with $|A|>\tan \theta_{i}$. Intuitively, this seems to confirm the idea that the tails become more and more stable, but unfortunately this idea cannot be made more precise within the present approach. For $\left(-1-\sin \theta_{i}\right) /\left(2 \cos \theta_{i}\right) \leq A$ $\leq\left(1-\sin \theta_{i}\right) /\left(2 \cos \theta_{i}\right)$, the $\lambda_{ \pm}$are real.

The constants $b$ and $c$ in (9.10) and (9.13) must be chosen so that Eq. (9.2) and (9.4) or (9.5) are satisfied at the interface. Using (9.6), Eqs. (9.4) and (9.5) imply $\delta \theta_{i}=-\delta \kappa / A$ so that (9.2) at the interface can be written

$$
\begin{aligned}
&\left(\frac{\partial \delta h_{1}}{\partial u_{1}}\right)_{i}-\left(\delta h_{1}\right)_{i}-\delta \kappa\left(1+\tan \theta_{i} / A\right) \\
&=\left(d_{0} / l\right) \delta \kappa\left(A \tan \theta_{i}+A^{2}\right)
\end{aligned}
$$

Evaluating (9.10) and (9.13) for $u_{1}=0$ and using (9.11) we find

$$
b=\frac{-\left(\frac{d_{0}}{l}\right) A^{2}(c+1)}{1+\left(\frac{d_{0}}{l}\right) A^{2}}
$$

Note that $b=0$ in the Ivantsov limit. Finally, from (9.5) we get

$$
c=-\frac{\left(\lambda_{-}-D\right)}{\left(\lambda_{+}-D\right)}
$$

where

$$
D=\frac{\tan \theta_{i} A+\frac{d_{0}}{l} A^{2}}{1+\frac{d_{0}}{l} A^{2}} .
$$

Thus we are able to satisfy the linearized equations and boundary conditions for any value of $A$. There are stable $(A>0)$ and unstable $(A<0)$ directions in an essentially infinite-dimensional phase space. The exponential behavior of the perturbations again illustrates why the simple truncations discussed in Sec. VII yield inconsistent and indeed contradictory results.

Finally, note that perturbations both to the Ivantsov and the non-Ivantsov solution behave in a similar manner. The non-Ivantsov term in the temperature equation adds a simple constant boundary term to Eq. (9.2). However, the additional integration constant $b$ in (9.13) allows (9.2) to be satisfied for nonzero $d_{0}$ with $b$ chosen as in (9.15). The overall description and flow in phase space is virtually identical for the two cases. 


\section{FINAL REMARKS}

Our analysis of the model equations has touched on several more general issues. We have argued on physical grounds that the tails of the steady-state needle crystal solutions can accommodate themselves to a variety of small perturbations near the tip. We will indeed show elsewhere ${ }^{19}$ by a mathematical analysis of the full problem that the dominant term in the tails has this property for distances larger than a diffusion length from the tip. In other words, the motion of the tip is not affected by asymptotic properties of the tails. Certainly it is hard to believe that features that manifest themselves only deep in the tails can be relevant for dendrite tip motion. Our discomfort with the microscopic-solvability scenario as applied to dendrites stems in part from the fact that the nontrivial "matching conditions" may be (and for the local models are) sensitive to just such details in the tails.

The above-mentioned asymptotic results for the "stability" of the tails for distances larger than the diffusion length $l_{t}$ go against the sensitivity seen in the local models. It is quite conceivable, though, that this result may be relevant only for relatively large Péclet numbers where the tip radius of curvature of the dendrite is of the same order as the diffusion length, since for small Péclet numbers such considerations would apply only very deep in the tails.

There are a number of physical effects that can change the asymptotic properties of needle solutions. For example, one can show that in the absence of interface kinetics [ $\mu=0$ in Eq. (1.2)] the asymptotic shape for Péclet number $p<\frac{1}{2}$ goes as $z(x)=-x^{2} / 2 p+a_{0} / x^{\alpha}$ with $\alpha>1$. On the other hand, for any nonzero $\mu$ we have $z(x)$ $=-x^{2} / 2 p+a_{1} x$ asymptotically. ${ }^{42}$ We believe asymptotic differences such as these have no physical significance, but they might affect the matching conditions.

An even more dramatic effect on the asymptotic steady-state shape is found if we add a phenomenological heat-loss term $-\Gamma T$ to the right-hand side of the heatconduction equation (1.1). This could be of some experimental relevance for crystal growth between two parallel plates as carried out by Honjo, Ohta, and Sawada, ${ }^{43}$ where $\Gamma$ takes account of heat loss to the plates. Very roughly speaking, a larger $\Gamma$ acts like a larger $\Delta$; both changes reduce the heat surplus. However, many features of steady-state propagation are very sensitive to $\Gamma$. For example, for $\Gamma>0$ and any $\Delta$, steady-state growth of a plane can occur at some (sufficiently small) velocity $V_{0}$, since the heat surplus can now be balanced by the heat-loss term. Presumably there also exist needlelike steady-state solutions ${ }^{44}$ moving at a larger velocity $V$ (depending on the curvature); for $\Gamma$ very small the shape must be essentially parabolic near the tip. However, deep in the tails, we no longer find $\theta \rightarrow \pi / 2$ as before. Rather the shape must approach that of a wedge, with a nonvertical angle $\theta_{0}$ satisfying $V \cos \theta_{0}=V_{0}$. It is important to see how the microscopic-solvability predictions are affected by changes such as these.

All this assumes we are in the regime of free dendritic growth, where side walls play no role, as is the case in most experiments. In the limit that the Péclet number $p \rightarrow 0^{+}$, the diffusion length becomes larger and larger and eventually effects of side walls must be taken into account. This is always the case for the Laplace (HeleShaw) limit. In the steady state, this implies an exponential approach of perturbations of the finger to its asymptotic shape, in contrast to the power-law behavior seen for a needle crystal. We have argued, mainly on physical grounds, that this difference may be crucial for a proper understanding of the needle crystal problem, at least at large Péclet numbers, and have shown that indications to the contrary from the model equations cannot be trusted. We hope that future analytical work will lead to a critical assessment of these ideas.

As presently formulated, the solvability condition seems open to such questions because of its close association with the physically unrealizable steady-state problem. A study of steady states certainly provides a great mathematical simplification, and it has demonstrated how subtle the mathematical mechanism that leads to selection could be. However, it seems to us that a deeper, and more physically accessible, understanding of dendritic growth is likely to be found in a dynamical study that deals directly with the physical entities, the dendrite tip, and the side branches, that are seen in the experiments. This may provide the needed matching of an intuitive picture with the new mathematical ideas. Since there have been so many revisions in our theoretical understanding of dendritic growth, we should be prepared for still more surprises to come.

\section{ACKNOWLEDGMENTS}

We are grateful to the participants at the 1986 workshop on pattern formation at the Aspen Center for Physics for a number of very helpful discussions.

\section{APPENDIX A: TREATMENT OF TIME-DEPENDENT CURVILINEAR COORDINATES}

In this appendix we discuss various definitions, identities, and kinematic relationships satisfied by our timedependent $2 d$ curvilinear coordinate system. Consider first a point on an arbitrary curve in the $x-z$ plane, specified by a position vector $\mathbf{r}$ from a space-fixed origin. The curvature $\kappa$ describes the rate of change of $\theta$, the angle between the normal to the curve and the (space-fixed) $z$ axis, with respect to a displacement $s$ along the curve. Our starting point is the geometrically obvious Frenet formu$\operatorname{las}^{45}$

$$
\frac{\partial \hat{\mathbf{n}}}{\partial s}=\kappa \hat{\tau} ; \quad \frac{\partial \widehat{\tau}}{\partial s}=-\kappa \hat{\mathbf{n}}
$$

where $\hat{\mathbf{n}}$ and $\hat{\boldsymbol{\tau}}$ are unit normal and tangent vectors to the curve.

Applying these to the $u_{j}$ curves $(j=1,2)$ in our orthogonal curvilinear coordinate system $\left(u_{1}, u_{2}\right)$, we have

$$
\frac{\partial \widehat{\mathbf{a}}_{i}}{\partial s_{j}}=\kappa_{i} \widehat{\mathbf{a}}_{j} ; \quad \frac{\partial \widehat{\mathbf{a}}_{i}}{\partial s_{i}}=-\kappa_{j} \widehat{\mathbf{a}}_{j}
$$

for $(i, j)=(1,2)$ with $(i \neq j)$. Here $\hat{\mathbf{a}}_{j}$ is the unit normal 
vector to the $u_{j}$ curve, $\kappa_{j}$ is the curvature, and the displacement $d s_{j}=h_{j} d u_{j}$ normal to the $u_{j}$ curve involves the scale factor ${ }^{36} h_{j}\left(u_{1}, u_{2}, t\right)$. Since $\partial s_{j} / \partial u_{j}=h_{j}$ we have $\partial f / \partial s_{j}=\left(1 / h_{j}\right)\left(\partial f / \partial u_{j}\right)$. Note that the equality of cross derivatives $\partial^{2} f / \partial u_{1} \partial u_{2}=\partial^{2} f / \partial u_{2} \partial u_{1}$ does not imply that $\partial^{2} f / \partial s_{1} \partial s_{2}=\partial^{2} f / \partial s_{2} \partial s_{1}$.

We have chosen sign conventions in defining the curvatures in (A1) and (A2) so that the curvature $\kappa_{1 i}$ of a convex protrusion of the solid into the liquid is positive. The arc length $s_{2}$ is measured from the $z$ axis along a $u_{1}$ curve while $s_{1}$ is measured from the interface along a $u_{2}$ curve. Finally, we define $\theta$ as the angle between the (outward) normal $\hat{\mathbf{a}}_{1}$ and the $z$ axis, and note that it satisfies $\cos \theta=\hat{\mathbf{z}} \cdot \hat{\mathbf{a}}_{1}$. See Fig. 6(b). It follows that $\kappa_{1}=\partial \theta / \partial s_{2}$ and $\kappa_{2}=-\partial \theta / \partial s_{1}$.

In terms of the position vector $\mathbf{r}$, we have

$$
\frac{\partial \widehat{\mathbf{r}}}{\partial u_{j}}=h_{j} \hat{\mathbf{a}}_{j}
$$

and the equality of cross derivatives $\partial^{2} \mathbf{r} / \partial u_{1} \partial u_{2}$ $=\partial^{2} \mathbf{r} / \partial u_{2} \partial u_{1}$, along with (A2) and (A3), yields the result

$$
\kappa_{i}=\frac{1}{h_{i} h_{j}} \frac{\partial h_{j}}{\partial u_{i}}=\frac{\partial \ln h_{j}}{\partial s_{i}} \quad(i \neq j) .
$$

A relationship between these curvatures follows from the equality of cross derivatives of $\widehat{\mathbf{a}}_{j}$ using (A2) and (A4),

$$
\frac{\partial \kappa_{1}}{\partial s_{1}}+\frac{\partial \kappa_{2}}{\partial s_{2}}=-\kappa_{1}^{2}-\kappa_{2}^{2}
$$

Next consider the time dependence of the coordinates. The normal velocities $V_{j}$ of the $u_{j}$ curves in the laboratory frame satisfy

$$
\left(\frac{\partial \mathbf{r}}{\partial t}\right]_{u} \equiv \mathbf{W}=V_{1} \hat{\mathbf{a}}_{1}+V_{2} \hat{\mathbf{a}}_{2} .
$$

Here the notation ()$_{u}$ indicates a derivative at constant $u_{1}$ and $u_{2}$. At constant $\mathbf{r}$ (i.e., a fixed point in the laboratory frame) we have the general chain-rule relation

$$
\left(\frac{\partial f}{\partial t}\right]_{\mathrm{r}}=\left[\frac{\partial f}{\partial t}\right]_{u}+\left(\frac{\partial f}{\partial u_{1}}\right)\left[\frac{\partial u_{1}}{\partial t}\right]_{\mathrm{r}}+\left(\frac{\partial f}{\partial u_{2}}\right)\left(\frac{\partial u_{2}}{\partial t}\right)_{\mathrm{r}} \text {. }
$$

Applying this to $f=\mathbf{r}$ we find from (A6) and (A3),

$$
V_{j}=-h_{j}\left(\frac{\partial u_{j}}{\partial t}\right)_{\mathbf{r}}
$$

and we note that (A7) can be rewritten in the compact form ${ }^{37}$

$$
\left[\frac{\partial f}{\partial t}\right]_{u}=\left[\frac{\partial f}{\partial t}\right]_{\mathrm{r}}+\mathbf{W} \cdot \nabla f
$$

Using the equality of cross derivatives $\partial^{2} \mathbf{r} /$ $\partial t \partial u_{j}=\partial^{2} \mathbf{r} / \partial u_{j} \partial t$, and (A3) and (A6), we find on taking components

$$
\frac{\partial V_{2}}{\partial s_{1}}-V_{1} \kappa_{2}=\frac{\partial \widehat{\mathbf{a}}_{1}}{\partial t} \cdot \hat{\mathbf{a}}_{2}=\left(\frac{\partial \theta}{\partial t}\right)_{u},
$$

$$
\begin{aligned}
& \frac{\partial V_{1}}{\partial s_{2}}-V_{2} \kappa_{1}=\frac{\partial \hat{\mathbf{a}}_{2}}{\partial t} \cdot \hat{\mathbf{a}}_{1}=-\left(\frac{\partial \theta}{\partial t}\right)_{u}, \\
& \frac{\partial V_{1}}{\partial s_{1}}+V_{2} \kappa_{2}=\left(\frac{\partial \ln h_{1}}{\partial t}\right)_{u}, \\
& \frac{\partial V_{2}}{\partial s_{2}}+V_{1} \kappa_{1}=\left(\frac{\partial \ln h_{2}}{\partial t}\right)_{u} .
\end{aligned}
$$

Only two of these, say (A10) and (A12), are independent since equality of the further cross derivatives with respect to $u_{i}$ or $u_{j}$ has already been assured by the symmetry leading to Eq. (A4). Two such independent equations can be found by adding (A10) to (A11), yielding

$$
\frac{\partial}{\partial u_{1}}\left(\frac{V_{2}}{h_{2}}\right)+\left(\frac{h_{1}}{h_{2}}\right)^{2} \frac{\partial}{\partial u_{2}}\left(\frac{V_{1}}{h_{1}}\right)=0,
$$

and adding (A12) to (A13), giving

$$
\frac{\partial}{\partial t}\left(h_{1} h_{2}\right)_{u}=\frac{\partial}{\partial u_{1}}\left(h_{2} V_{1}\right)+\frac{\partial}{\partial u_{2}}\left(h_{1} V_{2}\right) \text {. }
$$

Many other exact results can be found. Here we mention only the equation for the rate of change of $\kappa_{1}$, obtained by differentiating (A11) with respect to $u_{2}$ and using (A13),

$$
\left[\frac{\partial \kappa_{1}}{\partial t}\right]_{u}=-\left(\kappa_{1}^{2}+\frac{\partial^{2}}{\partial s_{2}^{2}}\right) V_{1}+V_{2} \frac{\partial \kappa_{1}}{\partial s_{2}}
$$

At the interface, using boundary conditions (4.2) and (4.3), this reduces to results given by Ben-Jacob et al. ${ }^{10}$ and Brower et al. ${ }^{9}$

If we consider a derivative at constant arc length $s_{2}$ and $u_{1}$ of the position vector $\mathbf{r}$, we find from the chain rule and (A3) and (A6)

$$
V_{1}=\left(\frac{\partial \mathbf{r}}{\partial t}\right)_{s_{2}} \cdot \hat{\mathbf{a}}_{1}
$$

and

$$
\left[\frac{\partial s_{2}}{\partial t}\right]_{u}=-h_{2}\left(\frac{\partial u_{2}}{\partial t}\right)_{s_{2}}=V_{2}-\hat{\mathbf{a}}_{2} \cdot\left(\frac{\partial \mathbf{r}}{\partial t}\right)_{s_{2}} .
$$

In general, evaluating (A17) and (A18) requires nonlocal information since $(\partial \mathrm{r} / \partial t)_{s_{2}}$ can be determined only if the behavior of the entire curve from the origin where $s_{2}=0$ is known. This can be seen explicitly by noting that the differential arc length $d s_{2}=h_{2} d u_{2}$ for fixed $d u_{2}$ varies in time because of the time dependence of $h_{2}$, so that $\partial\left(d s_{2}\right) / \partial t=\left(\partial \ln h_{2} / \partial t\right)_{u} d s_{2}$. Integrating over arc length and using (A13) we find the nonlocal representation

$$
\left(\frac{\partial s_{2}}{\partial t}\right)_{u}=V_{2}-V_{2}\left(s_{2}=0\right)+\int_{0}^{s_{2}} d s_{2}^{\prime} V_{1} \kappa_{1},
$$

which reduces at the interface to results found by Brower et al. ${ }^{9}$ and Ben-Jacob et al. ${ }^{10}$ As shown in Appendix C, these results simplify considerably in the steady state. 


\section{APPENDIX B: EVALUATION OF $(\partial H / \partial t)_{u}$}

The temperature equation in the laboratory frame is

$$
D \nabla^{2} T=\left(\frac{\partial T}{\partial t}\right]_{\mathbf{r}} .
$$

Using (A9) and expressing the Laplacian in curvilinear coordinates $^{36}$ we find

$$
\begin{aligned}
h_{1} h_{2}\left(\frac{\partial T}{\partial t}\right)_{u}= & h_{2} V_{1} \frac{\partial T}{\partial u_{1}}+h_{1} V_{2} \frac{\partial T}{\partial u_{2}} \\
& +D\left(\frac{\partial}{\partial u_{1}} \frac{h_{2}}{h_{1}} \frac{\partial}{\partial u_{1}}+\frac{\partial}{\partial u_{2}} \frac{h_{1}}{h_{2}} \frac{\partial}{\partial u_{2}}\right) T .
\end{aligned}
$$

(B2)
This will be useful in computing the derivative of $H$, given in Eq. (5.1) as

$$
H=\frac{1}{h_{2 i}} \int_{0}^{\infty} d u_{1} h_{1} h_{2} T
$$

Then

$$
\begin{aligned}
\left(\frac{\partial H}{\partial t}\right]_{u}= & -\left[\frac{\partial \ln h_{2 i}}{\partial t}\right]_{u} H+\frac{1}{h_{2 i}} \int_{0}^{\infty} d u_{1} h_{1} h_{2}\left(\frac{\partial T}{\partial t}\right)_{u} \\
& +\frac{1}{h_{2 i}} \int_{0}^{\infty} d u_{1} \frac{\partial}{\partial t}\left(h_{1} h_{2}\right)_{u} T
\end{aligned}
$$

Using (B2) and (A15), we have

$$
\begin{aligned}
{\left[\frac{\partial H}{\partial t}\right]_{u}=} & -\left(\frac{\partial \ln h_{2 i}}{\partial t}\right]_{u} H+\frac{1}{h_{2 i}} \int_{0}^{\infty} d u_{1} \frac{\partial}{\partial u_{1}}\left(h_{2} V_{1} T\right)+\frac{1}{h_{2 i}} \int_{0}^{\infty} d u_{1} \frac{\partial}{\partial u_{2}}\left(h_{1} V_{2} T\right) \\
& +\frac{D}{h_{2 i}} \int_{0}^{\infty} d u_{1} \frac{\partial}{\partial u_{1}}\left[\frac{h_{2}}{h_{1}} \frac{\partial T}{\partial u_{1}}\right)+\frac{D}{h_{2 i}} \int_{0}^{\infty} d u_{1} \frac{\partial}{\partial u_{2}}\left(\frac{h_{1}}{h_{2}} \frac{\partial T}{\partial u_{2}}\right) .
\end{aligned}
$$

Making use of the boundary conditions (4.2), (4.3), and (4.7), and using (A13), the first, second, and fourth terms on the right can be evaluated immediately and we find

$$
\begin{aligned}
{\left[\frac{\partial H}{\partial t}\right]_{u}=} & V_{n}\left(1-T_{i}\right)-H V_{n} \kappa_{1 i}+\frac{\partial}{\partial s_{2 i}} \int_{0}^{\infty} d s_{1} V_{2} T \\
& +D \frac{\partial}{\partial s_{2 i}} \int_{0}^{\infty} d s_{1} \frac{\partial T}{\partial s_{2}}
\end{aligned}
$$

which is Eq. (5.2).

Note that (B6) and all the other results in Appendixes $A$ and $B$ hold true independent of the particular choice we take for the coordinate system describing the temperature field away from the interface.

\section{APPENDIX C: STEADY-STATE RELATIONS}

Many results of Appendix A simplify greatly in the steady state, where we consider a needle in uniform translation for which $\left(\partial \mathbf{r}_{i} / \partial t\right)_{s}=V \hat{\mathbf{z}}$. Here $\hat{\mathbf{z}}$ is the unit vector in the $z$ direction. A position vector $\mathbf{R}$ whose origin moves with the tip of the needle satisfies $\mathbf{R}=\mathbf{r}-V t \widehat{\mathbf{z}}=\mathbf{r}-V t\left(\widehat{\mathbf{a}}_{1} \cos \theta-\widehat{\mathbf{a}}_{2} \sin \theta\right)$. From the chain rule relating $(\partial \mathrm{r} / \partial t)_{R}$ to $(\partial \mathrm{r} / \partial t)_{u}$ we find using $(\mathrm{A} 8)$

$$
-\left(\frac{\partial u_{1}}{\partial t}\right)_{\mathbf{R}}=\frac{V_{1}-V \cos \theta}{h_{1}}
$$

and

$$
-\left(\frac{\partial u_{2}}{\partial t}\right)_{\mathbf{R}}=\frac{V_{2}+V \sin \theta}{h_{2}} .
$$

In the steady state, physical quantities $f$ moving with the needle (e.g., the temperature field $T$ ) satisfy $(\partial f / \partial t)_{\mathbf{R}}=0$. Further, for steady states we can choose a coordinate system so that in the moving frame (i.e., at constant $\mathbf{R}$ ) the $u_{1}$ curves do not vary with time. This is obviously the case for the steady-state interface at $u_{1}=0$ and we use the freedom of choice of coordinates away from the interface to require it for all $u_{1}$ curves. The shape of all the orthogonal $u_{2}$ curves is then determined and the only time dependence left is the time-dependent value $u_{2}(t)$ we associate with each fixed $u_{2}$ curve. This remaining time dependence arises from the requirement that at the interface $V_{2 i}=0$. [Note from $(\mathrm{C} 2)$ that to have no time dependence of the $u_{2}$ coordinates in the moving frame, as is the case for the moving parabolic coordinates $(\xi, \eta)$ of Horvay and Cahn, ${ }^{7}$ requires that $V_{2 i}$ $=-V \sin \theta_{i}$.] Thus we can choose $\left(\partial u_{1} / \partial t\right)_{\mathbf{R}}=0$ for all $u_{1}$ and $\mathbf{R}$ and require that $\left(\partial u_{2} / \partial t\right)_{\mathbf{R}}$ be independent of $u_{1}$ for all $\mathbf{R}$ along a given $u_{2}$ curve. We note in particular that both coordinate systems (4.4) and (4.6) defined in terms of the temperature field are consistent with these choices. Equations $(\mathrm{C} 1)$ and $(\mathrm{C} 2)$ then reduce to

$$
\begin{aligned}
& V_{1}=V \cos \theta, \\
& \frac{V_{2}+V \sin \theta}{h_{2}}=\frac{V \sin \theta_{i}}{h_{2 i}},
\end{aligned}
$$

where the right-hand side of $(\mathrm{C} 4)$ is the value at the interface. Equations (C3) and (C4) give the steady-state integration of the general equations (A10)-(A13). Note that requiring $\left(\partial u_{1} / \partial t\right)_{R}=0$ for all $u_{1}$ implies that $(\partial \mathbf{r} / \partial t)_{s_{2}}=V \hat{\mathbf{z}}$. Thus $(\mathrm{A} 17)$ is equivalent to $(\mathrm{C} 3)$ and (A18) becomes in the steady state

$$
\left(\frac{\partial s_{2}}{\partial t}\right)_{u}=V_{2}+V \sin \theta
$$

Since $(\partial \mathbf{R} / \partial t)_{u}=(\partial \mathbf{r} / \partial t)_{u}-V \hat{\mathbf{Z}}$ we have, using (C3) and (C5), 


$$
\left(\frac{\partial \mathbf{R}}{\partial t}\right)_{u}=\widehat{\mathbf{a}}_{2}\left[\frac{\partial s_{2}}{\partial t}\right]_{u} .
$$

In the moving frame the velocity of a constant $\left(u_{1}, u_{2}\right)$ point given in (C6) is always tangential to the $u_{1}$ curve and has magnitude $V_{2}+V \sin \theta$. This contrasts to the laboratory-frame velocity $(\partial \mathrm{r} / \partial t)_{u}$, which at the interface is normal to the $u_{1}$ curve and which in general contains only the small $V_{2}$ tangential component.

Since physical quantities $f$ moving with the needle satisfy $(\partial f / \partial t)_{\mathbf{R}}=0$, we have using $(\mathrm{C5})$ the steady-state chain-rule result

$$
\begin{aligned}
{\left[\frac{\partial f}{\partial t}\right]_{u} } & =\left[\frac{\partial f}{\partial s_{2}}\right]\left(V_{2}+V \sin \theta\right) \\
& =\left[\frac{\partial f}{\partial u_{2}}\right]\left[\frac{V \sin \theta_{i}}{h_{2 i}}\right],
\end{aligned}
$$

where we have used (C4).

We can use these results to simplify Eq. (6.2). Using (C4) to obtain an expression for $h_{2} / h_{2 i}$ and substituting into $(5.1)$ we see that in the steady state

$$
H \sin \theta_{i}=M-\widetilde{J}_{\text {conv }},
$$

where $M$ is given in (6.3) and $\widetilde{J}_{\text {conv }} \equiv J_{\text {conv }} / V$ is given in (5.4). In this way we obtain (6.4).

\section{APPENDIX D: CURVILINEAR COORDINATE RELATIONS FROM THE TEMPERATURE EQUATION}

In this appendix we examine the equation relating the $h_{j}, \kappa_{j}$, and $V_{j}$ fields that result when we choose a particular coordinate system so that the exact temperature field is given by

$$
T=T_{i} e^{-u_{1}},
$$

as in (4.4). A similar analysis can be carried out for the choice (4.6). As discussed in Sec. IV B,

$$
\begin{aligned}
& T_{i}=\Delta-d_{0} \kappa, \\
& h_{1 i}=l,
\end{aligned}
$$

where we recall the notation $\kappa \equiv \kappa_{1 i}$ and $s \equiv s_{2 i}$. As in Eq. (B2) we note that that the temperature equation can be written as

$$
\begin{aligned}
\left(\frac{\partial T}{\partial t}\right]_{u}= & V_{1} \frac{\partial T}{\partial s_{1}}+V_{2} \frac{\partial T}{\partial s_{2}} \\
& +D\left[\kappa_{1} \frac{\partial}{\partial s_{1}}+\kappa_{2} \frac{\partial}{\partial s_{2}}+\frac{\partial^{2}}{\partial s_{1}^{2}}+\frac{\partial}{\partial s_{2}^{2}}\right] T
\end{aligned}
$$

Substituting (D1) and multiplying by $h_{1}^{2} / D T$ we find our basic result

$$
\begin{aligned}
\frac{\partial \ln h_{1}}{\partial u_{1}}-h_{1} \kappa_{1} & +1-\frac{h_{1} V_{1}}{D} \\
=\frac{h_{1}^{2} d_{0}}{T_{i}}[ & -\frac{1}{D}\left(\frac{\partial \kappa}{\partial t}\right)_{u}+\frac{V_{2}}{D}\left(\frac{h_{2 i}}{h_{2}}\right) \frac{\partial \kappa}{\partial s} \\
& \left.+\left(\frac{h_{2 i}}{h_{2}}\right) \frac{\partial^{2} \kappa}{\partial s^{2}}+\frac{\partial \kappa}{\partial s} \frac{\partial}{\partial s_{2}}\left(\frac{h_{2 i}}{h_{2}}\right)\right] .
\end{aligned}
$$

The $(\partial \kappa / \partial t)_{u}$ term can be found from Eq. (A16) evaluated at the interface. Note that the right-hand side of (D5) vanishes in the Ivantsov limit.

In the steady state (D5) becomes

$$
\begin{aligned}
\frac{\partial \ln h_{1}}{\partial u_{1}}-h_{1} \kappa_{1} & +1-\frac{h_{1} V \cos \theta}{D} \\
=\frac{h_{1}^{2} d_{0}}{T_{i}}[ & -\frac{\partial \kappa}{\partial s} \frac{V}{D} \sin \theta_{i}+\left(\frac{h_{2 i}}{h_{2}}\right) \frac{V_{2}}{D} \frac{\partial \kappa}{\partial s} \\
& \left.+\left(\frac{h_{2 i}}{h_{2}}\right) \frac{\partial^{2} \kappa}{\partial s^{2}}+\frac{\partial \kappa}{\partial s} \frac{\partial}{\partial s_{2}}\left(\frac{h_{2 i}}{h_{2}}\right)\right],
\end{aligned}
$$

where from (C4)

$$
\frac{h_{2 i}}{h_{2}}=\frac{V \sin \theta_{i}}{V_{2}+V \sin \theta}=1+O(1-\Delta) .
$$

The right side of (D6) is $O\left((1-\Delta)^{2}\right)$ for smooth solutions. If we make no smoothness assumptions about $\kappa$ derivatives and instead assume only that $l(\partial \kappa / \partial s)=O(\kappa l)$, etc., then to lowest order in $(1-\Delta)$, Eq. (D6) becomes

$$
\begin{aligned}
& \frac{\partial \ln h_{1}}{\partial u_{1}}-h_{1} \kappa_{1}+1-(V / D) h_{1} \cos \theta \\
& =d_{0}\left(-l \frac{\partial \kappa}{\partial s} \tan \theta_{i}+l^{2} \frac{\partial^{2} \kappa}{\partial s^{2}}\right)+O\left((1-\Delta)^{2}\right) .
\end{aligned}
$$

Equation (D8) is used in Sec. IX. Note that to $O(1-\Delta)$ the right side of (D8) is independent of $u_{1}$.

For simplicity, consider the Ivantsov limit, where (D8) becomes

$$
\frac{\partial \ln h_{1}}{\partial u_{1}}-h_{1} \kappa_{1}+1-\frac{V h_{1}}{D} \cos \theta=0 .
$$

At the interface we have $h_{1 i}=l=D \Delta /\left(V \cos \theta_{i}\right)$ and (D9) yields

$$
\left(\partial \ln h_{1} / \partial u_{1}\right)_{i}=\kappa l-(1-\Delta) .
$$

Further, we note that since $\kappa_{2}=\partial \ln h_{1} / \partial s_{2}$, we can write

$$
\kappa_{2 i}=\kappa \frac{\partial \ln l}{\partial \theta}=\kappa \tan \theta_{i} .
$$

Equation (D9) can be differentiated with respect to $u_{1}$ to yield the higher-order derivatives at the interface needed in the Taylor-series expansion. Thus the second-order term is

$$
\begin{aligned}
\frac{1}{h_{1}} \frac{\partial^{2} h_{1}}{\partial u_{1}^{2}}= & {\left[\frac{1}{h_{1}} \frac{\partial h_{1}}{\partial u_{1}}\right)^{2}+\frac{\partial h_{1}}{\partial u_{1}} \kappa_{1} } \\
& +h_{1} \frac{\partial \kappa_{1}}{\partial u_{1}}+\frac{V}{D} \frac{\partial}{\partial u_{1}}\left(h_{1} \cos \theta\right)
\end{aligned}
$$


We note that the $\partial \kappa_{1} / \partial u_{1}$ term can be evaluated at the interface using Eq. (A5). This term is clearly $O\left((1-\Delta)^{2}\right)$ for smooth solutions as are all other terms on the righthand side of (D12) with the exception of the last term, which may appear only first order in $(1-\Delta)$. However, working out the derivatives and evaluating at the interface we find

$$
\begin{aligned}
\frac{1}{l}\left(\frac{\partial^{2} h_{1}}{\partial u_{1}^{2}}\right)_{i}= & {[\kappa l-(1-\Delta)][2 \kappa l-(1-\Delta)] } \\
& +l\left(\frac{\partial \kappa_{1}}{\partial u_{1}}\right)_{i}+\Delta\left[\kappa l / \cos ^{2} \theta-(1-\Delta)\right]
\end{aligned}
$$

When $\kappa l$ in the last term is expressed in terms of $\theta$ as in Eq. (8.1), we note the last term is indeed $O\left((1-\Delta)^{2}\right)$ for the smooth Ivantsov solution. Higher-order derivatives of the $h_{1} \cos \theta$ term in (D9) behave in a similar manner.

\section{APPENDIX E: MODEL EQUATIONS}

We analyze the CFM where

$$
H=T_{i} l(1+a \kappa l) \text {, }
$$

with $a>0$, and $J_{\text {cond }}$ and $J_{\text {conv }}$ as in the BLM, as discussed in Sec. VII. For $a=0$, we have the BLM of BenJacob et al. ${ }^{10}$

We first consider the Ivantsov limit where $T_{i}=\Delta$. It is clear from (6.2) that with $a>0$ in (E1) there is a twodimensional $(\theta, \kappa)$ phase space. To obtain the phase flow, we consider $\kappa$ as a function of $\theta$, noting that $\partial / \partial s=\kappa \partial / \partial \theta$, and (6.2) becomes

$a \sin \theta_{i} \widetilde{\kappa} \frac{\partial \widetilde{\kappa}}{\partial \theta_{i}}=\left[\frac{\cos ^{3} \theta_{i}(1-\Delta)}{\Delta^{2}}-\widetilde{\kappa}-a \Delta \widetilde{\kappa}^{2}\left(\frac{1+\sin ^{2} \theta_{i}}{\cos \theta_{i}}\right)\right]$,

where $\widetilde{\kappa} \equiv D \kappa / V$.

First consider behavior in the tails near the (Ivantsov) fixed point $\widetilde{\kappa}=\partial \widetilde{\kappa} / \partial \theta=0$ at $\theta_{i}=\pi / 2$. Let $\omega \equiv \pi / 2-\theta_{i}$, and keep low-order terms in $\omega$ in (E2),

$$
\begin{aligned}
-a \tilde{\kappa}(\partial \widetilde{\kappa} / \partial \omega)= & \left(\omega^{3}+\frac{1}{2} \omega^{5}+\cdots\right) \\
& \times(1-\Delta) / \Delta^{2}-\widetilde{\kappa}-2 a \Delta \widetilde{\kappa}^{2} / \omega .
\end{aligned}
$$

There is a power-series solution $\bar{\kappa}$ of (E3) flowing into the fixed point $\omega=0$ satisfying

$$
\bar{\kappa}=\omega^{3}(1-\Delta) / \Delta^{2}+O\left(\omega^{5}\right),
$$

and a recursion relation can be derived for the higherorder terms. Note that $\bar{\kappa}$ becomes parabolic for small $\omega$ and that the coefficient of the $\omega^{3}$ term agrees with the exact Ivantsov parabola to first order in $(1-\Delta)$. However, $\bar{\kappa}$ does not necessarily satisfy the smoothness condition at the tip $\partial \bar{\kappa} / \partial \theta=0$. To obtain an acceptable needle crystal solution we examine the phase-space structure around $\bar{\kappa}$ in the tails ${ }^{46}$ by writing

$$
\widetilde{\kappa}=\bar{\kappa}+\epsilon,
$$

and linearizing in $\epsilon$. We find from (E3) to lowest order

$$
-a(1-\Delta) \omega^{3} \partial \epsilon / \partial \omega=-\epsilon,
$$

or

$$
\epsilon \sim \exp \left\{-1 /\left[2 a(1-\Delta) \omega^{2}\right]\right\} .
$$

Thus the fixed point in the tails is stable and we should be able to use a component of the latter solution to satisfy both $\partial \widetilde{\kappa} / \partial \theta=0$ at the tip and still flow to the Ivantsov fixed point in the tails. We can verify that there is only one trajectory satisfying $\partial \kappa / \partial \theta=0$ at the tip. This condition implies that the right-hand side of (E2) must vanish for $\theta=0$ and uniquely fixes the tip curvature as

$$
\begin{aligned}
\widetilde{\kappa}_{t} & =[-1+\sqrt{1+4(1-\Delta) a / \Delta}] / 2 a \Delta \\
& \approx(1-\Delta)+O\left((1-\Delta)^{2}\right),
\end{aligned}
$$

which agrees with the exact Ivantsov result to $O(1-\Delta)$. Note that in Eqs. (E2) and (E6), one can consider $a$ as a "singular perturbation."

The results of this counting argument are fully confirmed by a numerical solution of (E2). The CFM has a family of solutions in the Ivantsov limit for all $\Delta$ and reduces to a parabolic shape in the tails. It reproduces the exact Ivantsov parabola to first order in $(1-\Delta)$. A flow diagram is given in Fig. 7.

Next consider the general case with $d_{0} \neq 0$. There now is a three-dimensional phase space and (6.2) becomes for $\widetilde{\kappa}$ as a function of $\theta_{i}$,

$$
\begin{aligned}
\widetilde{d}_{0} T_{i} \cos \theta_{i} \widetilde{\kappa}^{2} \widetilde{\kappa}^{\prime \prime}= & \cos ^{3} \theta_{i}\left(1-T_{i}\right)-T_{i}^{2} \widetilde{\kappa}-a \widetilde{\kappa}^{3} T_{i}^{3}\left(1+\sin \theta_{i}\right) / \cos \theta_{i} \\
& -\widetilde{\kappa} \widetilde{\kappa}^{\prime} \sin \theta_{i} T_{i}\left[a T_{i}^{2}-\widetilde{d}_{0}\left(3 T_{i} a \widetilde{\kappa}+2 \cos \theta_{i}-\widetilde{\kappa}\right)\right]+\widetilde{d}_{0}^{2} \widetilde{\kappa}^{2}\left(\widetilde{\kappa}^{\prime}\right)^{2} \cos \theta_{i}-\widetilde{d}_{0} T_{i} \cos \theta_{i} \widetilde{\kappa}\left(\widetilde{\kappa}^{\prime}\right)^{2},
\end{aligned}
$$

where $\kappa^{\prime}=\partial \kappa / \partial \theta$, etc., and $\widetilde{d}_{0} \equiv d_{0} V / D$. For $a=0$ this reduces to the BLM, Eq. (7.2).

We analyze $(\mathrm{E} 10)$ to lowest order in $(1-\Delta)$ noting that $T_{i} \equiv 1+O(1-\Delta)$. As before, linearizing about the smooth solution $\bar{\kappa}$ that satisfies (E10) in the tails, with $\pi / 2-\theta_{i}=\omega$, and keeping the most significant terms, we find

$$
\epsilon^{\prime \prime}-\frac{a \epsilon^{\prime}}{d_{0}(1-\Delta) \omega^{4}}+\frac{\epsilon}{d_{0}(1-\Delta)^{2} \omega^{7}}=0,
$$

where $\epsilon^{\prime}=\partial \epsilon / \partial \omega$, etc. Here $\omega=0$ is an irregular singular point, and as usual we can obtain the controlling factor ${ }^{29}$ by the substitution

$$
\epsilon \equiv e^{A} \text {. }
$$

Ignoring the result $A^{\prime \prime}$ term (which will turn out to be consistent as $\omega \rightarrow 0$ ), we obtain from (E11) a quadratic equation for $A^{\prime}$, whose solutions as $\omega \rightarrow 0$ yield

$$
A_{+} \sim \frac{-a}{3 d_{0}(1-\Delta) \omega^{3}}
$$




$$
A_{-} \sim \frac{-1}{2 a(1-\Delta) \omega^{2}} .
$$

The second root (E14) is independent of $d_{0}$ and indeed is the Ivantsov-like solution (E7). Both trajectories very rapidly approach $\bar{\kappa}$; the tails in this model are completely attractive. We thus expect and do find numerically a family of solutions-indeed we find a "family of families" since there is a range of $\kappa_{t}$ with $\kappa_{t}^{\prime}=0$ for which solutions can be found. The flow in phase space is very contractive, as illustrated in Fig. 8 and discussed in Sec. VII.

It is instructive to contrast this behavior with that of the BLM with $a=0$. Then the equivalent of Eq. (E11) becomes

$$
\epsilon^{\prime \prime}+\frac{2 \epsilon^{\prime}}{\omega^{3}(1-\Delta)}+\frac{\epsilon}{d_{0}(1-\Delta)^{2} \omega^{7}}=0 .
$$

The sign of the $\epsilon^{\prime}$ term has changed and there is no $d_{0}$ factor. Solutions of the form of (E12) as $\omega \rightarrow 0$ are

$$
A_{ \pm}=\frac{1 \omega^{-2}}{2(1-\Delta)} \pm \frac{2 i \omega^{-5 / 2}}{5 \sqrt{d_{0}}} \text {. }
$$

Both these trajectories oscillate and spiral away from $\bar{\kappa}$ as $\omega \rightarrow 0$, and in general we expect a solvability condition. Equation (E16) is consistent with the WKB analysis of the BLM given by Langer. ${ }^{47}$

Finally, we mention an attempt to somewhat systematically generate a model equation. The $J_{\text {cond }}$ term in the BLM is $O\left((1-\Delta)^{2}\right)$ for smooth solutions using the coordinate system (4.4). This suggests that we should expand the $\left(H \sin \theta_{i}+\widetilde{J}_{\text {conv }}\right)$ terms in $(6.2)$ or, equivalently, $M$ in
(6.4), to $O\left((1-\Delta)^{2}\right)$ as in Sec. IX, using the results in Appendix $D$. Of course, the expansion makes sense only for the smooth solutions, so the approach is not really systematic in general, and cannot serve as a controlled way to derive models. We find that the first-order $\kappa l$ correction to $H$ mentioned in Sec. VII is canceled by a term from $J_{\text {conv }}$, but that a similar term along with a $\partial \kappa / \partial s$ term is found at $O\left((1-\Delta)^{2}\right)$ for $d_{0}=0$. Thus we can provide no systematic mathematical justification for the CFM but derivative terms do appear at every order.

If we restrict ourselves to models in a three-dimensional phase space, then it turns out, as argued in Sec. II B, that the Ivantsov derivative terms dominate all others. Thus we can simply consider the Ivantsov limit $d_{0}=0$. The resulting second-order equation is most compactly written in terms of the variable $\widehat{\kappa} \equiv \widetilde{\kappa} / \cos ^{3} \theta$ as

$$
\begin{aligned}
& \beta \widehat{\boldsymbol{\kappa}}+\widehat{\boldsymbol{\kappa}}^{2}+\sin \theta \cos \theta \hat{\boldsymbol{\kappa}} \frac{\partial \widehat{\boldsymbol{\kappa}}}{\partial \theta} \\
&-\cos ^{2} \theta \widehat{\boldsymbol{\kappa}} \frac{\partial}{\partial \theta}\left[\widehat{\boldsymbol{\kappa}} \frac{\partial \widehat{\boldsymbol{\kappa}}}{\partial \theta} \cos ^{2} \theta\right]=\frac{(1-\Delta)}{\Delta^{4}},
\end{aligned}
$$

where $\beta \equiv 1+(1-\Delta)^{2} / \Delta^{2}$. The exact Ivantsov solution has $\hat{\boldsymbol{\kappa}}=$ const and (E17) has a solution $\bar{\kappa}$ that tends to this in the tails. Analysis like that done before shows there are one stable and one unstable additional direction, appropriate for the existence of a (simple) family of solutions. The structure of this equation is unchanged if a second derivative term from $J_{\text {cond }}$ is also considered. In fact, we find that the most important non-Ivantsov corrections come from $H$, rather than from $J_{\text {cond }}$.
${ }^{1}$ A standard review is J. S. Langer, Rev. Mod. Phys. 52, 1 (1980). For a review of more recent work, see J. S. Langer, Institute of Theoretical Physics (Santa Barbara, CA) Report No. ITP-85-79 (unpublished); and D. A. Kessler, J. Koplik, and $\mathrm{H}$. Levine (unpublished).

${ }^{2}$ C. Huang and M. E. Glicksman, Acta Metall. 29, 701 (1981); 29, 717 (1981).

${ }^{3}$ For a recent review, see M. E. Glicksman, Mat. Sci. Eng. 61, 45 (1984).

${ }^{4}$ It was traditionally thought that these simplifications retain most essential features of the full problem. However, if the "microscopic-solvability picture," (discussed later in this section) turns out to be correct, then dendritic growth depends on anisotropy and possibly other details of the system, and there could be qualitatively different predictions in two dimensions (2D) or in 3D. However, most of the important conceptual and mathematical issues are present in the $2 \mathrm{D}$ one-sided limit, and it allows immediate contact with the model equations.

${ }^{5}$ Again this might alter conclusions in $2 \mathrm{D}$ because the asymptotic shape of steady-state needles is very different; the curvature term drops off as $x^{-3}$ while the undercooling due to interface kinetics only falls off as $V \cos \theta \sim 1 / x$. In 3D, both terms go as $r^{-1}$. However, present indications are that interface kinetics does not affect the solvability condition [C. Caroli (private communication)].

${ }^{6}$ G. P. Ivantsov, Dokl. Acad. Nauk SSSR 58, 567 (1947).
${ }^{7}$ G. Horvay and J. W. Cahn, Acta Metall. 9, 695 (1961).

8 J. S. Langer and H. Müller-Krumbhaar, Acta Metall. 26, 1681; 26, 1689 (1978); 26, 1697 (1978).

${ }^{9}$ R. Brower, D. Kessler, J. Koplik, and H. Levine, Phys. Rev. Lett. 51, 1111 (1983); Phys. Rev. A 29, 1335 (1984); D. A. Kessler, J. Koplik, and H. Levine, ibid. 30, 2860 (1984); 30, 3161 (1984); 31, 1713 (1985); 34, 4980 (1986).

${ }^{10}$ E. Ben-Jacob, N. D. Goldenfeld, J. S. Langer, and G. Schön, Phys. Rev. Lett. 51, 1930 (1983); Phys. Rev. A 29, 330 (1984); E. Ben-Jacob, N. D. Goldenfeld, B. G. Kotliar, and J. S. Langer, Phys. Rev. Lett. 53, 2120 (1984).

${ }^{11}$ Both the capillary length $d_{0}$ and the kinetic coefficient $\mu$ in Eq. (1.3) are affected by crystalline anisotropy. Recent experiments on real $3 d$ systems [M. Glicksman (private communication); see also J. S. Langer, 1986 Les Houches Lectures (unpublished)] do not seem to show much dependence of the dendritic growth rate on crystalline anisotropy, but a more complete analysis is needed to provide a definitive comparison between experiment and theory on the role of anisotropy.

${ }^{12}$ B. I. Shraiman, Phys. Rev. Lett. 56, 2028 (1986).

${ }^{13}$ R. Combescot, T. Dombre, V. Hakim, Y. Pomeau, and A. Pumir, Phys. Rev. Lett. 56, 2036 (1986).

${ }^{14}$ D. C. Hong and J. S. Langer, Phys. Rev. Lett. 56, 2032 (1986).

${ }^{15}$ A. Barbieri, D. C. Hong, and J. S. Langer, Phys. Rev. A 35, 1802 (1987).

${ }^{16}$ D. Meiron, Phys. Rev. A 33, 2704 (1986). 
${ }^{17}$ D. Kessler, J. Koplik, and H. Levine, Phys. Rev. A 33, 3352 (1986).

${ }^{18} \mathrm{~A}$ preliminary account of this work has been published. See W. van Saarloos and J. D. Weeks, Phys. Rev. Lett. 55, 1685 (1985).

${ }^{19}$ W. van Saarloos and J. D. Weeks, Phys. Rev. A 35. 2357 (1987).

${ }^{20}$ The inclusion of the factor $T_{i}$ in the definition of $l$ is somewhat arbitrary, but it is used in the BLM and it simplifies some results [e.g., (4.8)] in the exact boundary-layer formulation to follow. We generally consider large undercooling with $T_{i}$ near unity.

${ }^{21}$ W. van Saarloos and J. D. Weeks, Phys. Rev. Lett. 51, 1046 (1983).

${ }^{22}$ W. W. Mullins and R. F. Sekerka, J. Appl. Phys. 34, 323 (1963); 35, 444 (1964).

${ }^{23}$ The instability is cut off at short wavelengths by surfacetension effects arising from the Gibbs-Thomson boundary condition (1.3). The scale of the resulting pattern is of order the geometric mean of the macroscopic diffusion length $2 D / V$ and the capillary length $d_{0}$. See Ref. 1 .

${ }^{24}$ Again this statement must be modified for needles so sharp that surface-tension effects become important.

${ }^{25}$ For a general review, see D. Bensimon, L. P. Kadanoff, S. Liang, B. I. Shraiman, and C. Tang, Rev. Mod. Phys. 58, 977 (1986).

${ }^{26}$ See P. M. Morse and H. Feshbach, Methods of Theoretical Physics (McGraw Hill, New York, 1953), Part 1, pp. 171-174 for a discussion of diffusion from this point of view.

${ }^{27}$ For further discussion, see M. Grant, W. van Saarloos, and J. D. Weeks (unpublished). D. A. Kessler, J. Koplik, and H. Levine, Phys. Rev. A 34, 4980 (1986) have numerically studied the diffusion equation in the presence of side walls. If there are different regimes, it is natural to expect a crossover when the diffusion length is of the order of the side-wall spacing. However, their results do not support this idea; rather the selection seems to be unaffected by the side-wall spacing. See Sec. III for a discussion of the numerical methods.

${ }^{28}$ See, e.g., R. Pieters and J. S. Langer, Phys. Rev. Lett. 56, 1948 (1986).

${ }^{29}$ For a general discussion, see C. M. Bender and S. A. Orszag, Advanced Mathematical Methods for Scientists and Engineers (McGraw-Hill, New York, 1978).

${ }^{30}$ Note that it is crucial in this argument that we consider free dendritic growth for which $l$ can become large, either because $V$ is small or because $\cos \theta$ tends to zero in the tails. In a Hele-Shaw cell the relevant macroscopic length-the distance of the pattern from the side wall-always remains finite and a similar straightforward ordering of derivatives does not occur. Indeed in some limits one can derive equations for the HeleShaw system resembling the local model equations in which $d_{0}$ appears in front of the highest derivative and is a singular perturbation. See M. Grant, W. van Saarloos, and J. D. Weeks (unpublished).

${ }^{31}$ D. Kessler, J. Koplik, and H. Levine, Phys. Rev. A 30, 2820 (1984).

${ }^{32}$ See the discussion in Ref. 27.

${ }^{33}$ P. Pelcé and Y. Pomeau, Stud. Appl. Math. 74, 245 (1986).

${ }^{34}$ It is necessary to consider time-dependent coordinates even for steady-state motion if we want to make contact with the model equations and require that $\left(\partial \mathbf{r}_{i} / \partial t\right)_{u_{2}}$ have only a normal component. Earlier, N. Goldenfeld (unpublished) discussed the formulation of a boundary-layer model using a different set of time-independent curvilinear coordinates.

${ }^{35}$ For an arbitrary time-dependent interface shape with concave as well as convex regions, the resulting coordinate system may be ill-defined in some regions. As in the BLM, we concentrate on slowlying varying and mainly convex time-dependent interface shapes or on the steady-state needle shapes where no such problems arise.

${ }^{36}$ For an elementary discussion see F. B. Hildebrand, Advanced Calculus for Applications (Prentice-Hall, New Jersey, 1962). The (diagonal) metric tensor elements $g_{i i}$ satisfy $g_{i i}=h_{i}^{2}$.

${ }^{37}$ For related work, see D. Bedeaux, A. M. Albano, and P. Mazur, Physica 82A, 438 (1976).

${ }^{38}$ Equation (6.2) can be formally integrated in terms of the function $x_{i}\left(\theta_{i}\right)$ giving the $x$ coordinate of the interface as a function of $\theta_{i}$, since $\cos \theta_{i}=\partial x_{i} / \partial s$. We find $\left(H-d_{0}\right) \sin \theta_{i}$ $+\widetilde{J}_{\text {conv }}+\widetilde{J}_{\text {cond }}=(1-\Delta) x_{i}\left(\theta_{i}\right)$.

${ }^{39} \mathrm{~A}$ similar point was made by B. Caroli, C. Caroli, B. Roulet, and J. S. Langer, Phys. Rev. A 33, 442 (1986), and J. Phys. (Paris) 47, 623 (1986) though the implications for the validity of the model equations were not explored. $(1-\Delta)$ is a singular perturbation in the particular truncation generating the BLM. See J. S. Langer and D. Hong, Phys. Rev. A 34, 1462 (1986) for a careful analysis of the BLM both for small and large undercoolings.

${ }^{40}$ Some (nonessential) terms were omitted in Eq. (8) of Ref. 18.

${ }^{41}$ A similar effect was pointed out by J. W. McLean and P. G. Saffman, J. Fluid Mech. 102, 455 (1981). See Sec. VIII.

${ }^{42}$ W. van Saarloos, J. D. Weeks, and B. G. Kotliar, Phys. Rev. A 35, 2288 (1987).

${ }^{43}$ E. Honjo, S. Ohta, and Y. Sawada, Phys. Rev. Lett. 55, 841 (1985).

${ }^{44}$ Indeed the "outer expansion model," discussed in Ref. 19, can be modified to include $\Gamma$, and has such a family.

${ }^{45}$ See F. B. Hildebrand, Ref. 36., pp. 271-275.

${ }^{46}$ Note that we linearize about $\bar{\kappa}$ rather than the Ivantsov solution, to which $\bar{\kappa}$ reduces as $d_{0} \rightarrow 0$.

47J. S. Langer, Phys. Rev. A 33, 435 (1986). 